\title{
EXCITONS AND ENERGY TRANSFER IN INSULATORS
}

J. Jortner ${ }^{\mathrm{a}}$, E.E. Koch ${ }^{\mathrm{b}}, \mathrm{N} \cdot$ Schwentner $^{\mathrm{c}}$

\footnotetext{
Department of Chemistry, Tel Aviv University, $69978 \mathrm{Tel}$ Aviv, Israel

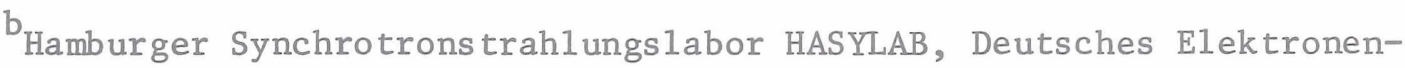
Synchrotron DESY, 2000 Hamburg 52, BRD

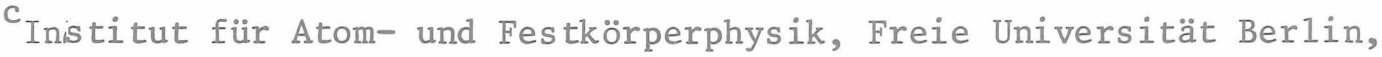
1000 Berlin, BRD
}

ABSTRACT

We review the current "state of art" of the understanding of excited-state energetics and dynamics of electronic excitations in large-gap insulators explored in the energy range 6-50 eV. We shall emphasize the interrelationsship between the molecular approach and the solid-state approach for the elucidation of the nature of these electronic excitations and their nonradiative relaxation in solids, liquids and dense fluids.

To appear in

Proceedings of the NATO Advanced Study Institute on Photophysics and Photochemistry in the Vacuum U1traviolet

Lake Geneva, Wisconsin 16-27 August 1982

(D. Reidel Publishing Co., Dordrecht, Holland, 1983) 



\section{PROLOGUE}

We shall be concerned with the electronic level structure and the excited-state nonradiative relaxation phenomena in molecular solids ${ }^{1}$ in the energy range 6-50 eV. Figure 1 provides an overview of the energy regions for the basic excitations and radiationless decay channels in molecular solids. Phonons, vibrational and librational excitations mark the lower energy limit at $0.01 \mathrm{eV}$. The elementary excitations at higher energies involve intervalence and low-intravalence electronic excitations, which can be described in terms of the tight-binding Frenkel scheme, $2-4$ as well as Wannier exciton ${ }^{5}$ and impurity states. At even higher energies one encounters interband transitions and impurity ionization states.

Dynamical processes in electronically excited states ${ }^{6}$ involve a variety of intrastate and interstate relaxation phenomena, e.g., exciton trapping via vibrational relaxation, electronic relaxation of exciton states manifested by interconversion of electronic energy to phonons, electronic energy transfer, autoionization of metastable excitons located above the band gap, electron-hole recombination and charge transfer processes. In what follows we shall dwell on electronic excitations in insulators in the energy range 6-50 eV, focusing attention on the following features:

(1) Nature of the electronic excitations in molecular solids, liquids and fluids.

(2) The relation between the electronic level structure and the geometrical structure of the condensed phase, emphasizing the effects of structural and compositional disorder on the electronic excitations.

(3) The parentage problem. The interrelationship between the electronicvibrational excitations of the constituents and the excitations in the condensed 
(4) Nonradiative relaxation of electronic excitations via intrastate nuclear deformation and interstate electronic relaxation.

(5) Influence of the state of aggregation on nonradiative relaxation processes.

(6) Electronic energy transfer phenomena, which pertain to basic issues of coherence and localization.

The present discussion is selective rather than exhaustive. A recent extensive review ${ }^{1}$ of the electronic excitations in condensed rare gases was provided by us. Our major goal will be to focus on the relations and correlations between the molecular approach and the solid-state approach for the elucidation of the excited-state energetics and dynamics of electronic excitations in insulators.

\section{FRENKEL EXCITONS}

These electronic excitations in pure molecular crystals have a definite parentage in an intravalence molecular (or atomic) excitation of the constituents. 2-5 Typical examples involve exciton states originating from $\pi \longrightarrow \pi$ * transitions in organic crystals, ${ }^{3,4}$ with which we shall not be concerned here, and the lowest electronic excitations in simple molectular crystals, ${ }^{7-11} \mathrm{e.g.,}$ solid $\mathrm{CO}$ and $\mathrm{N}_{2}$, which are located in the VUV range and which are of considerable interest for the present discussion. The description of the intermolecular interactions is considerably simplified as the intravalence excitations are characterized by a minor expansion of the excited-state charge distribution, whereupon intermolecular overlap corrections are negligible. Accordingly, the 
tight binding approximation is applicable for the description of exciton states. ${ }^{2-5}$ The crystal Hamiltonian is

$$
H=\sum_{n} \sum_{\alpha} H_{n \alpha}+\frac{1}{2} \sum_{n} \sum_{m} \sum_{\alpha} \sum_{\beta} V_{n \alpha, m \beta}
$$

where $H_{n \alpha}$ is the single-molecule Hamiltonian for a molecule located at the site $\alpha(\alpha=1 \ldots \sigma)$ in the nth unit cell $(n=1 . . N)$, while $V_{n \alpha, m \beta}$ denotes the intermolecular interaction. The single-molecule wave functions $\phi_{n_{\alpha}}^{0}$ and $\phi_{n_{\alpha}}^{f}$ for the ground electronic state $(0)$ and for the electronically excited state (f), respectively, can be utilized to construct the excitation-transfer integrals

$$
M_{n \alpha, m \beta}^{f}=\int\left(A \phi_{n \alpha}^{0} \phi_{m \beta}^{f}\right) v_{n \alpha, m \beta} \phi_{n \alpha}^{f} \phi_{m \beta}^{0} d t
$$

where $A$ is the intermolecular electron antisymmetrization operator. ${ }^{4}$ Following the conventional tight-binding approach, the lattice sums for the $f$ excited state are

$$
L_{\alpha \beta}^{f}(\underset{\sim}{k})=\sum_{n} e^{i \underset{\sim}{k} \cdot \underset{\sim}{n}} M_{n \alpha, m \beta}^{f}
$$

where $k$ is the lattice quasimomentum. The $\sigma \times \sigma$ energy matrix is

$$
\underset{\sim \alpha \beta}{H}(\underset{\sim}{k})=\left[\epsilon^{f}+D^{f}+L_{\alpha \alpha}^{f}(k)\right] \delta_{\alpha \beta}+L_{\alpha \beta}^{f}(k)\left(1-\delta_{\alpha \beta}\right)
$$

where $\epsilon^{f}$ is the isolated-molecule excitation energy, while $D^{f}$ is the environmental shift term consisting of electrostatic and dispersive interactions. Diagonalization of this energy matrix results in the energies $E_{j}(\underset{\sim}{k})(j=1 \ldots \sigma)$, which determine the exciton dispersion curves and yield the exciton density of states. As is well known, ${ }^{2-5}$ the momentum conservation rule implies that the 
exciton states amenable to optical excitation from the ground state of the crystal are characterized by $\underset{\sim}{k}=\underset{\sim}{Q}$, where $\underset{\sim}{Q}$ is the wavevector of exciting light, i.e., $|Q|=2 \pi / \lambda \approx 0$. The $k \approx 0$ optically accessible exciton states for a crystal containing two molecules per unit cell $(\sigma=2)$ are characterized by the energies

$$
\begin{aligned}
& E_{1}=\epsilon^{f}+D^{f}+L_{11}^{f}(\underline{k}=0)+L_{12}^{f}(\underline{k}=0) \\
& E_{2}=\epsilon^{f}+D^{f}+L_{11}^{f}(\underline{k}=0)-L_{12}^{f}(\underline{k}=0)
\end{aligned}
$$

The following physical implications of the tight-binding approach to exciton states are noteworthy.

(1) Davydov splitting. The optical spectra exhibit the celebrated Davydov splitting, which for a crystal with $\sigma=2$ is given by $\Delta \mathrm{E}_{\text {DAVYDOV }}=2 \mathrm{~L}_{12}(\underset{\sim}{\mathrm{k}}=0)$, reflecting the coupling between translationally unequivalent molecules.

(2) Weak exciton-vibron coupling. ${ }^{3,4}$ When the Davydov splitting is low relative to the intramolecular vibrational frequency; the excitation transfer integrals and the lattice sums are modified by the nuclear vibrational overlap Franck-Condon factors $\langle F C\rangle$, being given by $L_{\alpha \beta}(\underset{\sim}{k}=0)=L_{\alpha \beta}^{f}(\underset{\sim}{k}=0)\langle F C\rangle$, where $L_{\alpha \beta}^{f}$ is the electronic contribution, Eq. (2.3). This effective reduction of the electronic contribution amounts to a polaron effect. ${ }^{6}$ In this limit the Davydov splitting is proportional to the intensity of each vibronic component.

(3) Strong exciton-vibron coupling. ${ }^{3,4}$ When the Davydov splitting exceeds the intramolecular frequency, the excitation transfer occurs at a frozen nuclear configuration. The lattice sums are determined by the "bare" excitation transfer integrals, i.e., $L_{\alpha \beta}(k=0)=L_{\alpha \beta}(k=0)$.

(4) Solvent shifts. The energetic shift of the center of gravity of the manifold of the Davydov components relative to the excitation energy of the bare molecule is given by ${ }^{2} \Delta E_{s}=D^{f}+L_{\alpha \alpha}(\underset{\sim}{k}=0)$, being determined by the environmental 
shift term, as well as by the resonance interaction with translationally equivalent molecules. In the weak coupling limit the contribution of $\mathrm{L}_{\alpha \alpha}$ to each $\Delta \mathrm{E}_{\mathrm{S}}$ is determined by the relative intensity of the particular vibronic component.

(5) Crystal-field mixing. The first-order scheme outlined above requires extension, going beyond a single electronically excited configuration. Off-resonance interactions, induced by the crystal Hamiltonian (2.1), with high electronically excited states can readily be incorporated in the tight-binding scheme by extending the matrix $(2.4)$ to include several electronic states. ${ }^{2-4}$ Crystal-field mixing will result in gross modifications of the absolute intensities, as well as of the relative intensities of the Davydov components. The most dramatic effect of crystal-field mixing pertains to the appearance of the electronic origin of crystal excitons, which have a parentage in symmetryforbidden molecular transitions. These electronic excitations become allowed, in principle, in the crystal, whose Hamiltonian is of. lower symmetry, being induced by the crystal Hamiltonian stealing intensity from higher, allowed, first-order exciton states.

(6) Longitudinal and transverse excitons. When the exciton band structure is determined by dipole-dipole interactions, the dipole lattice sums, Eq. (2.3), are piecewise discontinuous at $\underset{\sim}{k} \rightarrow 0 .^{12}$ For an exciton state which has its parentage in a triple degenerate $p$ atomic state, two types of $\underset{\sim}{k} \simeq 0$ exciton states will be exhibited, ${ }^{5,13}$ i.e., longitudinal excitons with $\underset{\sim}{\mu}|| \underset{\sim}{\mathbf{~}}$, and transverse excitons with $\underset{\sim}{\mu} \perp \underset{\sim}{\mathrm{k}}$, where $\underset{\sim}{\mu}$ is the transition moment. The longitudinal-transverse splitting is $4 \pi \rho|\underset{\sim}{\mu}|^{2}$, where $\rho$ is the molecular density. For optical excitations in an ideal crystal with $\underset{\sim}{k}=\underset{\sim}{Q}$, only the transverse excitons are allowed; ${ }^{5,13}$ however, exciton scattering at imperfections will result in the appearance of the bncitudinal exciton branch in the optical spectrum. 
(7) Cooperative electronic-vibrational excitons. 14-16 Up to this point we have been concerned with electronic-vibrational excitations located on a single molecule, which in the solid-state language are referred to as "one particle" excitations. One can construct "two-particle" electronic-vibrational excitations where the electronic excitation and the vibrational excitation reside on different molecules. In view of the frequency difference between the ground and electronically excited states, the one-particle and the two-particle excitations will appear at different energies. The two-particle excitation is optically forbidden in zero order, but excitonic interactions result in mixing between the one-particle and the two-particle states. As the total momentum ${\underset{\sim}{t}}_{t}$ of the twoparticle states $\underset{\sim}{\mathrm{k}} \underset{\mathrm{f}}{\mathrm{k}}=\underset{\sim}{\mathrm{k}}+\underset{\sim}{\mathrm{k}} \mathrm{v}$ is a superposition of the exciton momentum $\underset{\sim}{\mathrm{k}}$ and the vibron momentum $\underset{\sim}{k}$, then the conservation rule for optical excitation $\underset{\sim}{k_{t}}=\underset{q}{q}$ results in excitation with $\underset{\sim}{\mathrm{k}}=-\underset{\sim}{\mathrm{k}}$, whereupon two-particle exciton states with all $\underset{\sim}{k}$ values will appear in the optical crystal spectrum, providing central information on the exciton density of states. 15,16

The experimental implications of Frenkel excitons for the elucidation of the VUV spectra of simple molecular crystals below the conduction band are of considerable interest. ${ }^{7-11}$ Figure 2 shows the absorption spectrum of crystalline CO in the energy range $7.5-10.0 \mathrm{eV}$, which exhibits electronic-vibrational Frenkel excitons, originating from the $X^{\gamma} \Sigma^{+} \rightarrow A^{1}$ II allowed transition of the co molecule. The vibrational spacings considerably exceed the intermolecular interactions, so that the excitonic states correspond to the weak coupling situation. The vibronic exciton with $v=1,2$ and 3 exhibit a definite splitting, which may tentatively be attributed to Davydov splitting in the crystal containing two molecules per unit ce11. The appearance of the Davydov splitting for the most intense vibronic components of the excitonic transition is borne out by the theoretical description 
of the weak exciton-vibron coupling situation. It should be noted, however, that the double hump structure exhibited for these intense vibronic components may originate from phonon side bands ${ }^{17}$ and further studies are required to resolve the issue of whether excitonic splitting is indeed exhibited in the first singlet exciton state of solid CO. The $\mathrm{N}_{2}$ molecule is isoelectronic with CO and the corresponding first spin-allowed molecular excitation is the $x^{1} \sum_{g}^{+} \rightarrow a^{\lambda} \Pi_{g}$ transition, which is symmetry forbidden in the $\mathrm{N}_{2}$ molecule. Crystal-field mixing makes this transition partly allowed in solid $\mathbb{N}_{2}$, as is apparent from Fig. 3. No Davydov splitting seems to be exhibited for the vibronic components of this excitonic transition. Similarly, the second spin-allowed excitonic transition of solid $\mathrm{N}_{2}$ in the energy range 9-12 eV (Fig. 3), which has its parentage in the symmetry forbidden $x^{1} \sum_{g}^{+} \rightarrow w^{1} \Delta_{w}$ molecular excitation, is induced in the crystal by crystal-field mixing. The third Frenkel exciton state of solid $N_{2}$, which originates from the symmetry allowed $x^{1} \sum_{g} \rightarrow b^{1} \Pi_{u}$ molecular transition, appears in the $\mathrm{N}_{2}$ crystal in the energy range 12-14 eV (Fig. 4). This excitonic transition is characterized by quite large dipoledipole coupling, which contribute to the intermolecular excitation-transfer integrals. The intense vibronic components exhibit the Davydov splitting (Fig. 4), as is expected for the weak coupling situation. Up to this point we have been concerned with one-particle excitations in solid $\mathrm{CO}$ and $\mathrm{N}_{2}$. The twoparticle excitations in these crystals are characterized by two features. Firstly, they are well separated in energy, e.g., the frequency change between the $x^{1} \Sigma_{g}$ and $\mathrm{a}^{1} \mathrm{II}_{\mathrm{g}}$ states of $\mathrm{N}_{2}$ is $\sim 500 \mathrm{~cm}^{-1}$. Secondly, in view of the large energy spacing between the two-particle excitation and the corresponding one-particle state, the intensity of the former is low. Figure 5 shows the weak two-particle excitations 
in solid $N_{2}$, which originate from the $x^{1} \sum_{g}^{+} \rightarrow a^{1} \Pi_{g}$ and $x^{1} \sum_{g}^{+} \rightarrow w^{1} \Delta_{u}$ molecular transitions. A careful Comparison between the lineshapes of some conventional one-particle and the corresponding two-particle excitations (Fig. 6) reveal that the sideband is broader in the latter case, exhibiting the effect of the excitation of all the states in the exciton band for the two-particle cooperative excitations.

The one-particle and the two-particle Frenkel excitations in solid CO and $\mathrm{N}_{2}$ have a definite parentage in electronic excitations of a single molecule, constituting stable crystal excitations below the interband transition to the conduction band. An interesting example for stable exciton states was observed by Lau, Fock, Koch ${ }^{18,19}$ for solid $\mathrm{CO}_{2}$ in the energy range 8-13 eV. Figure 7 shows the absorption spectrum together with the photoelectron yield curves in solid $\mathrm{CO}_{2}$ in the energy range 8-30 eV. From the comparison between absorption and photoelectron yield curves (Fig. 7), it is apparent that the absorption spectrum above $15 \mathrm{eV}$ corresponds to interband transitions. The structure in the absorption spectrum below the onset of interband transitions at $13 \mathrm{eV}$ corresponds to stable exciton states. The sharp peak at $11 \mathrm{eV}$ in solid $\mathrm{CO}_{2}$ constitutes a stable exciton. Searching for a molecular parentage of this exciton state, we assign this exciton to a Frenkel-type exciton which has its parentage in the excited molecular states of $\mathrm{CO}_{2} 20$. One of them, the ${ }^{3} \Sigma_{u}$ state which is located at $11.2 \mathrm{eV}$ in the isolated molecule, has recently been shown to be essentially a Rydbery state with a substantial ( 30\%) intravalence character. ${ }^{20}$ Thus, the $11 \mathrm{eV}$ exciton state in solid $\mathrm{CO}_{2}$ should be considered as an intermediate exciton, which can be envisaged as a Frenkel exciton with substantial nonorthogonality corrections. 21,22 An alternative description of this exciton state, which has its parentage in a molecular Rydberg state, rests on a modified Wannier scheme. 21,22 This brings us to consider Wannier states in molecular crystals. 


\section{WANNIER EXCITONS}

The traditional description of Wannier excitons ${ }^{5}$ considers bound states in the band gap due to electron-hole attractive Coulomb interaction, which do not have parentage in the electronic excitations of the constituents (Fig. 8). The traditional approach to Wannier states rests on:

(A) The conventional effective mass approximation (EMA). ${ }^{23}$ This approach considers a single pair of valence and conduction bands, while the effect of higher transitions is subsummed into the dielectric constant, which screens the electron-hole Coulomb interaction. Next, and most important, the EMA rests on the cardinal assumption that the spatial extent of the electronic wavefunction is large relative to the lattice constant $r_{c}$. In a crystal, where the conduction band is parabolic, the EMA results in a hydrogenic wave equation for the envelope function $F(r)$ and the Wannier states

$$
\left[-\frac{h^{2}}{2 \mu} \nabla^{2}-\frac{e^{2}}{\epsilon r}\right] F(r)=E F(r)
$$

where $\epsilon$ is the (static) dielectric constant and $\mu$ is the exciton effective mass, i.e., $\mu^{-1}=m_{e}^{-1}+m_{h}^{-1}$. Equation (3.1) results in a hydrogenic series of Wannier excitations, which are characterized by the principal quantum number $n=1,2 \ldots$ (and angular momentum quantum number $\ell$ ) and whose energies relative to the bottom of the conduction band are

$$
E_{n}=-\mu e^{2} / 2 \epsilon^{2} h^{2} n^{2}
$$

while the radii of these states are

$$
r_{n}=n^{2} n^{2} \in / \mu e^{2}
$$


The radius of the lowest $n=1$ exciton state is $r^{n=1}=n^{2} \in / \mu e^{2}$, whereupon the validity condition for the applicability of the EMA is

$$
r^{n=1} \gg r_{c}
$$

An interesting class of simple molecular crystals, where a search for Wannier states can be conducted, involves rare-gas solids (RGS). As is apparent from Table I, for RGS $r^{n=1}<r_{c}$ and the EMA is expected to be inapplicable, whereupon this theoretical scheme predicts that in these molecular crystals, which are characterized by low dielectric constants, Wannier excitons will not be exhibited. Contrary to these theoretical considerations, which rest on a conventional solid-state approach, experimental studies pioneered by Schnepp and Dressler ${ }^{24}$ and by Baldini ${ }^{25}$ have established that RGS exhibit a rich spectrum of Wannier excitons. 26-28 From the spectra portrayed in Figs. 9 and 10, it is apparent that the low-lying electronic excitations of solid $\mathrm{Ne}, \mathrm{Ar}, \mathrm{Kr}$ and $\mathrm{Xe}$ reveal a long series of Wannier excitons with principal quantum number $n$ up to $n=5$. The general features of these electronic spectra of RGS are:

(1) The appearance of Wannier states with $n=2-5$. These "new" states have no apparent atomic parentage.

(2) The energies of these "new" states obey the hydrogenic relation, Eq. (3 2), with $n=2,3, \ldots$ etc.

(3) The "new" states exhibit spin-orbit splitting.

(4) The lowest electronic excitation is accompanied by a second intense transition separated from it by the spin-orbit coupling, $\Delta \mathrm{E}_{S O}$. The two intense transitions are labelled $n=1$ and $n^{\prime}=1$ in Figs. 9 and 10. These two intermediate excitons can be described in terms of two alternative pictures.

(4a) $n=1$ Wannier states subjected to large central cell corrections. 
(4b) Frenkel excitons with parentage in the ${ }^{1} S_{0} \rightarrow{ }^{3} P_{1}$ and ${ }^{1} S_{0} \rightarrow{ }^{1} P_{1}$ atomic excitations modified by large nonorthogonality corrections.

The phenomenology of the energetics of deep Wannier excitons in SRG (Fig. 8) rests on the hydrogenic level structures

$$
E_{n}=E_{G}-\frac{B}{n^{2}}
$$

where $E_{G}$ is the gap energy, while $B$ represents the exciton binding energy

$$
B=\mu e^{4} / 2 \epsilon^{2} h^{2}
$$

These exciton states involve the excitations of an electron from the top of the valence band to a bound state below the bottom of the conduction band (Fig. 8). Some of the features of the valence and conduction bands in RGS are summarized in Fig. 11.

The energetic relation (3.4) applies well to states with $n>1$, resulting in the estimates of $E_{G}$ and $B$ for RGS (Table II). The values of $B$, together with the experimental data for the dielectric constant $\epsilon$, result in the evaluation of the exciton effective mass $u$ according to Eq. (3.5). On the other hand, Eq. (3.4) fails miserably for the lowest $n=1$ exciton states in RGS. Adhering to the Wannier model we can assert that in this case the central cell correction ${ }^{29-31}$

$$
\Delta=E^{n=1}(\exp t)-E^{n=1}=E^{n=1}(\exp t)-\left(E_{G}-B\right)
$$

is substantial.

This success of the phenomenological description raises the intriguing question why, in spite of the failure of EMA, does the Wannier model work for RGS. In this context, a unified approach to deep Wannier excitons will be desirable. Several theoretical models were advanced in this context. 
(B) Pseudopotential theory. Phillips 31 has considered contributions to central cell corrections for the energies of deep Wannier excitons, which originate from the following sources: (i) Spatial dispersion of the effective mass. (ii) Spatial dispersion of the dielectric constant. (iii) Core nonorthogonality corrections. This approach suggests the use of a model potential, 29-31 which is Coulombic at large distances and constant for small distances from the positive ion core.

(C) The integral equation method. Andreoni et a1. 32,33 has adopted a Green's function approach with a local perturbation potential to account for the energies of Wannier states.

(D) The quantum defect method (QDM). This approach ${ }^{34-36}$ is of considerable interest in the context of the present discussion as it bridges the gap between atomic (molecular) excitations and solid-state large-radius exciton states for all values of $n$. Starting from the one-electron picture for deep Wannier excitons

$$
\left[-\frac{\hbar^{2}}{2 \mu} \nabla^{2}+U(r)\right] \psi(r)=E \psi(r)
$$

where the asymptotic form of the potential is

$$
U(r) \stackrel{r \rightarrow \infty}{\longrightarrow}-\frac{e^{2}}{\in r}
$$

The energy levels can be written in the form

$$
E_{n}=E_{G}-\frac{B}{\left(n+\delta_{n}\right)^{2}}
$$

where $n$ is the principal quantum number, while $\delta_{n}$ is the quantum defect, which depends on $n$. Table III provides a comparison jetween experimental transition energies for bulk excitons in solid neon and derived parareters 27 with theoretical calculations. 
for Wannier excitons

in terms of the QDM. It is interesting to

note that the $\delta_{n}$ values exhibit a very weak dependence on $n$. A heuristic approach to the QDM rests on the use of a model potential $v_{n}(r)$, which is different for each principal quantum number $n$, whose explicit form is 36

$$
\begin{aligned}
& V_{n}(r)=-C_{n} ; r<\rho_{n} \\
& V_{n}(r)=-\frac{e^{2}}{\epsilon r} ; r>\rho_{n},
\end{aligned}
$$

being constant for small distances and Coulombic for large $r$. The advancement of the model potential (3.10) is in the spirit of the pseudopotential theory (B). The Schrobdinger equation is now

$$
\left[-\frac{\hbar^{2}}{2 \mu} \nabla^{2}+V_{n}(r)\right] \psi_{n}=\left(E_{G}-E_{n}\right) \psi_{n}
$$

with the boundary condition $\psi_{n}(r) \stackrel{r \rightarrow \infty}{\longrightarrow} 0$, which makes it possible to solve this equation by equating the logarithmic derivatives of $\psi_{n}$ at $r=\rho_{n}$. The input data for the solution of the QDM equations (3.10) and (3.11) for RGS are of two types: (a) Atomic parameters. The energies for the excitations of the rare-gas atoms can be utilized for the calculation of the potential parameters $C_{n}$ and $\rho_{n}$. (b) Solid-state parameters $\mu, \in$ and $E_{G}$. The QDM equation is then solved to yield the quantum defects $\delta_{n}$, the energies $E_{n}$. Eq. (3.9), and the relative intensities $I_{n} \propto\left(n+\delta_{n}\right)^{-3}$. The practical advantages of the QDM scheme can be summarized as follows:

1) This scheme is applicable for valence excitations ${ }^{34}$ and for core excitations $^{35}$ in RGS, as well as for atomic impurity states.

2) The energies of deep Wannier exciton states, including the problematic $\mathrm{n}=1$ state are properly accounted for. 
The practical disadvantages of this scheme are:

3) Proliferation of parameters.

4) Relative intensities of the Wannier states are not properly accounted for. 28

In spite of these technical shortcomings, (3) and (4), the QDM is of considerable interest from the viewpoint of general methodology because of the following reasons:

5) It establishes the atomic parentage of Wannier states with high $n$.

6) It is capable of dealing, in principle, with the transition from the Frenkel (atomic) to the wannier (solid-state) picture.

Before concluding the discussion of deep Wannier exxciton states in RGS, it is appropriate to point out that with the increase of experimental sophistication of sample preparations and of spectroscopic techniques, new types of electronic excitations in films of solid rare gases were observed (Figs. 10 and 12). These fall into two categories:

[1] Surface Wannier exciton states with $n=1$ and $n=2$ were observed in thin films. ${ }^{28}$ (Figs. 10 and 12).

[2] The appearance of transverse-longitudinal exciton splitting was recorded $1,26,28$. While in an ideal solid in optical absorption only iransverse excitons appear, scattering of excitons by the surface and by imperfections yields a finite intensity to longitudinal excitons. The transverse-longitudinal exciton splittings in RGS summarized in Table IV yields some information concerning the strength of resonance coupling in RGS. 


\section{DEEP WANNIER ATOMIC IMPURITY STATES}

Rare-gas guest atoms in a solid rare-gas host provide the best known example of deep impurity states in insulators. Absorption spectra of these diluent raregas alloys were studied by Baldini and Knox, ${ }^{37}$ Baldini, ${ }^{38}$ Gedanken et al. ${ }^{39}$ and Pudewill et al., 40 among others. The central result of these spectroscopic studies is the appearance of a long Wannier series containing lines up to $n=5$ (Fig. 13). In each dilute alloy two distinct series are exhibited that are split by the spin-orbit interaction and converge to the bottom of the conduction band of the host matrix (Fig. 8). The energy levels of these deep Wannier impurity states with $n \geqslant 2$ obey the hydrogenic equation

$$
E_{n}^{i}=E_{G}^{i}-\frac{B^{i}}{n^{2}}
$$

where $E_{G}^{i}$ is the impurity gap, i.e., the impurity ionization potential in the solid, while

$$
B^{i}=\frac{\mu e^{e^{4}}}{2 \epsilon^{2} h^{2}}
$$

is the exciton binding energy, which is analogous to Eq. (3.5), with the exciton effective mass being replaced by the electron mass, $\mu_{e}$, in the conduction band. As is apparent from Fig. 14, the Wannier formula, Eq. (4.1), is extremely well obeyed for deep impurity states in solid $\mathrm{Ne}$. The values of $\mathrm{B}^{i}$ obtained for different impurities are independent of the nature of the impurity, being also close to the value of $B$ for pure $N e$, as $\mu_{e} \cong \mu$. On the other hand, the energy gaps derived from Fig. 14 depend on the nature of the impurity, as is expected. The values of $E_{G}^{i}$ and $B^{i}$ obtained from optical absorption spectroscopy are summarized in Table $V$. Supplementary information on $E_{G}^{i}$ (and $E_{G}$ ) can be obtained from 
the threshold for internal photoconductivity. Such data are not yet available for RGS, while for liquid rare gases this information has been obtained. 41 Novel information on the energetics of both exciton and impurity states originate from the threshold for photoemission $E_{T h}$ and $E_{T h}^{1}$ (Fig. 8), which are given by

$$
\begin{aligned}
& E_{T H}=E_{G}+V_{0} \\
& E_{T h}^{i}=E_{G}^{i}+V_{0}
\end{aligned}
$$

where $V_{0}$ is the energy of an electron at the botton of the conduction band relative to the vacuum level.Fig. 15 demonstrates the determination of the $\mathrm{E}_{\mathrm{Th}}^{i}$ values for imipurity states in RGS. These experimental data for photoemission thresholds are summarized in Table IV.

We are now in a position to provide a quantitative discussion of impurity ionization processes in solids, liquids and dense fluids. The impurity ionization potential in a condensed phase is given by ${ }^{42}$

$$
E_{G}^{i}=I_{G A S}+P_{+}^{i}+V_{0}
$$

where I GAS is the gas-phase ionization potential, while $P_{+}^{i}$ represents the medium polarization energy induced by the impurity. From Eqs. (4.4) and (4.5), we immediately infer that the threshold for photoemission from an impurity state is

$$
E_{T h}^{i}=I_{G A S}+P_{+}^{i}
$$

For doped solid rare gases the combination of $E_{G}^{j}$ data from absorption spectroscopy with photoemission yield data resulted in the determination of $P_{+}^{i}$ values and, more important, the establishment of $V_{0}$ values for RGS (Table VI). Extensive data are available on $V_{0}$ values in liquid rare gases ${ }^{1,43}$. A theoretical description 
advanced by Springett, Jortner and Cohen ${ }^{44}$ considers two major cumulative contributions to $v_{0}$, i.e.,

$$
V_{0}=T+U_{p},
$$

originating from short-range repulsive interactions, $T$, which are subsummed into a kinetic energy term, and background polarization energy $U_{p}$ (Table VI). From this analysis of the $V_{0}$ scale, it is apparent that a uniform description is provided for the energetics of an electron in the conduction band, both in a solid and in a liquid. Accordingly, we are led to the important conclusion that the notion of the conduction band is applicable to liquids and other structurally disordered dense fluids, so that there is no need to invoke the notion of translational symmetry for the description of this basic universal property of electronic states of condensed phases. 45

\section{EXCITONS AND IMPURITY STATES IN RARE-GAS FLUIDS}

The applicability of the universal concept of the conduction band to liquid rare gases raises the interesting question as to whether solid-state-type excitations, such as excitons and impurity states, are amenable to experimental observation in structurally disordered materials. Obviously, Frenkel-type states, which have a unique parentage in the states of the constituents, will definitely be observed. What is more interesting to inquire is whether Wannier exciton and impurity states do "exist" in dense fluids. On the basis of theoretical arguments, it was conjectured ${ }^{46}$ that large-radius Wannier excitons are amenable to experimental observation in a dense fluid, where electron-medium interaction is sufficiently weak. Liquid rare gases constitute a medium where electron-atom interaction is moderately weak, ensuring that the linewidth of Wannier states is not excessive. Some experimental work has established that $n=2$ and $n=3$ Wannier excitons 47,48 and $n=2$ Wannier 
impurity states ${ }^{49,50}$ are amenable to experimental observation in liquid rare gases.

In order to establish the nature of Wannier states in simple dense fluids, it is interesting to obtain a description of the electronic excitations of a guest atom or molecule in a host fluid, where the fluid density is continuously varied over a broad density range. ${ }^{50-52}$ In this context three types of electronic transitions of the guest atom should be considered, involving (a) intravalence excitations, (b) low extravalence excitations, which are accompanied by a change $\Delta n_{a}=1$ in the principal quantum number $n_{a}$, and (c) high extravalence excitations characterized by $\Delta \mathrm{n}_{\mathrm{a}}=2$. Figures 16 and 17 show the absorption spectra of Xe-Ar, spanning a broad density range of the Ar host, from the atomic limit through the liquid to the solid. In the energy range 9.5-10.8 eV, the following five transitions can be identified.

Transition (I) to $6 s^{1}[1 / 2] J=1\left(5 p^{5}\left\{^{2} P_{1 / 2}\right\} 6 s\right)$ at $9.57 \mathrm{eV}(1296 \AA)$
Transition (II) to $5 d[1 / 2] J=1\left(5 p^{5}\left\{^{2} P_{3 / 2}\right\} 5 d\right)$ at $9.92 \mathrm{eV}(1250 \AA)$
Transition (III) to $5 d[5 / 2] J=3\left(5 p^{5}\left\{^{2} P_{3 / 2}\right\} 5 d\right)$ at $10.25 \mathrm{eV}(1210 \AA)$,
which is symmetry-forbidden in the atomic limit

Transition (IV) to $5 d[3 / 2] J=1\left(5 p^{5}\left\{{ }^{2} P_{3 / 2}\right\} 5 d\right)$ at $10.40 \mathrm{eV}(1192 \AA)$

Transition (V) to $7 \mathrm{~s}[3 / 2] \mathrm{J}=1\left(5 \mathrm{p}^{5}\left\{{ }^{2} \mathrm{P}_{3 / 2}\right\} \mathrm{ss}\right)$ at $10.60 \mathrm{eV}(1170 \AA)$

Transitions (II)-(IV) are due to intravalence excitations. Transition (I) corresponds to a low extravalence excitation with $\Delta n_{a}=$ l of a Rydberg series which starts with the first resonance line $6 s[3 / 2] \mathrm{J}=1\left(5 \mathrm{p}^{5}\left\{{ }^{2} \mathrm{p}_{3 / 2}\right\} 6 \mathrm{~s}\right)$ at $8.44 \mathrm{eV}(1470 \AA$ A). Transition (V) constitutes a high extravalence excitation with $\Delta n_{a}=2$. The following medium effects have been observed at low and moderate densities (Fig. 16). 
(1) Satellite bands. In the density range $0.1-0.4 \mathrm{gcm}^{-3}$ a single blue satellite band accompanies each of the transitions (I) and (IV). The intensity at these blue satellites increases with increasing density until at Ar density $\approx 0.6 \mathrm{gcm}^{-3}$ they merge together with the corresponding resonance line into a single band.

(2) Symmetry breaking effects. The transition (III) to a $\mathrm{J}=3$ state is symmetry-forbidden in the atomic limit, as is evident from Fig. 16, being induced by the host fluid.

(3) Medium-induced shifts of the lowest $\Delta n_{a}=1$ extravalence excitations. These shifts are summarized in Fig. 18. The Xe resonance lines $6 \mathrm{~s}$ [3/2] (not shown in Figs. 16-18) and 6s' [1/2] (Transition I) are weakly red-shifted at low densities, exhibiting a large blue shift at higher densities. A similar behavior is observed for Transition (IV). These features have been explained in terms of a strongly repulsive Xe-Ar interaction potential in the excited state. ${ }^{50-52}$

(4) Medium-induced shifts of the higher $\Delta n_{a}=2$ extravalence excitation. The spectral shift of Transition (V) to the 7s [3/2] state drastically differs from those revealed by the $6 s[3 / 2]$ and $6 s^{\prime}[1 / 2]$ transitions. While the latter low extravalence excitations exhibit predominantly a large spectral blue shift (Fig. 18), the former "high" extravalence transition reveals an appreciable spectral red shift even at high densities. In the high density range (Fig. 17), the first transition at $\approx 9.7 \mathrm{eV}\left(1265-1290 \AA\right.$ ) was assigned by Messing et al. ${ }^{50}$ to an excitation of the $6 s^{\prime}[1 / 2]$ state (Transition I) and the third main transition at $\approx 10.5 \mathrm{eV}(1170-1190 \AA$ ) to the $5 d[3 / 2]$ state (Transition IV). These transitions exhibit large blue shifts (Figs. 17) at high densities. The most interesting feature of the spectra in Fig.17 is the absorption band at 9.9-10.2 eV. This second band centered at $10.12 \mathrm{eV}$ $(1225 \AA)$ in the high density fluid was assigned by Messing et al. 50 to an $n=2$ Wannier state on the basis of the following arguments. Firstly, this transition is close in 
energy to the $n=2$ Wannier impurity state in solid Xe/Ar alloys. Secondly, the intensity of this absorption in the Xe/Ar system at high (> $7 \times 10^{-3}$ atoms $\AA^{-3}$ ) densities is anomalously enhanced, indicating the appearance of a new absorption band at these densities. This behavior is unique to the Xe/Ar system and was not observed for $\mathrm{Xe} / \mathrm{Ne}$ and $\mathrm{Xe} / \mathrm{He}$ in the same range of atomic densities.

The assignment of the $n=2$ [3/2] Wannier impurity state of Xe in Ar fluid makes it possible to establish a correlation between atomic states and Wannier excitations. Figure 19 provides a correlation diagram advanced by Messing et al. ${ }^{50}$ which relates the high extravalence atomic excitations and "high" $n=2$ states. From this analysis of the spectroscopy of impurity states in a dense fluid, the following conclusions emerge :

1) There exists a possible parentage of high $n=2$ Wannier states in high $\Delta n_{a}=2$ extravalence atomic excitations of the constituent observation. This calls for an extension of the QDM approach to impurity states in fluids.

2) Both s and d atomic impurity states were observed in the dense fluid.

3) Metastable impurity states, e.g., ${ }^{2} P_{3 / 2}(5 d) \mathrm{J}=1$ excitation of $X e$, were observed above the onset of the conduction band. ${ }^{31}$ The absorption lineshapes for such autoionizing states should exhibit Fano resonances. ${ }^{53}$ However, these interesting effects may be masked by phonon broadening and inhomogeneous broadening phenomena.

\section{MOLECULAR WANNIER IMPURITY STATES IN SOLID RARE GASES}

The observation of intermediate and Wannier-type atomic impurity states in RGS and in fluids implies that analogous molecular excitations should be amenable to experimental observation. There has been a lively controversy as to whether molecular Rydberg states "exist" in solid matrices. 22,54-56 This question should be paraphrased 
to inquire, "what are the relevant matrix effects which will prohibit the spectroscopic detection of molecular extravalence excitations in simple solids". 22 Extensive experimental work, ${ }^{22,39}$ some of which is presented in Figs. 20 and 21, has established that intermediate-type and higher Wannier-type molecular excitations are observed in RGS. The following features of molecular Rydberg states in RGS should be emphasized:

(1) The first molecular extravalence excitation corresponds to an intermediate case, which can be described either in terms of the first molecular Rydberg state subjected to large nonorthogonality corrections or, alternatively, to a $n=$ ] Wannier state with large central cell corrections. The former description implies large blue spectral shifts of the lowest molecular Rydberg state in the matrix (Table VII). Furthermore, extensive line broadening is exhibited (Figs. 20 and 21 and Table VIII), originating from phonon broadening and also from some inhomogeneous broadening effects. We note in passing that the diagnostic criterion advanced by Robin 54,55 for the identification of molecular Rydberg states should be modified from "Molecular Rydberg states disappear in a condensed phase" to read, "The lowest molecular Rydberg transition is considerably blue shifted in a condensed phase".

(2) Higher Rydberg states, corresponding to a $n=2$ Wannier state of an electron bound to a positive ion in an RGS, were observed. $22,39,56$

(3) Vibrational structure for all $n$ molecular intermediate and Wannier-type excitations was observed. $22,39,56$ This new spectroscopic feature of molecular extravalence excitations in a dense medium corresponds to vibrational excitations of the positive ion core.

(4) Overlap of the molecular Wannier-type excitations with other broad structureless transitions, which correspond to dissociative states and which complicate the observation of molecular extravalence excitations in condensed phases. 
(5) The spectroscopic identification of the high molecular Wannier states provides a direct method for the determination of the molecular ionization potential in the solid, Eqs. (4.1) and (4.5), and the threshold $\mathrm{E}_{\mathrm{Th}}^{\mathrm{i}}$ for photoemission, Eq. (4.6). The $\mathrm{E}_{\mathrm{Th}}^{\mathrm{i}}$ values were determined directly by photoemission ${ }^{57,58}$ from molecular impurity states in RGS, being in good agreement with the spectroscopic data. 22,39 The consistency obtained between the spectroscopic and photoemission data provides strong support for the identification of molecular Wannier states in RGS.

Up to this point we were concerned with molecular Wannier impurity states in RGS. It is interesting to inquire whether Wannier exciton states can be observed in pure molecular crystals. The absorption spectrum of the acetylene crysta ${ }^{59}$ (Fig. 22) reveals two transitions: (a) The intravalence $\pi \rightarrow \Pi^{*}$ transition in the range 6.8-7.8 eV. The vibrational structure of this transition shows an extensive progression of the $2 v_{4}$ twisting vibration $\left(472 \mathrm{~cm}^{-1}\right.$ in the gas phase). This transition reveals a modest medium spectral shift, being red shifted by $0.1 \mathrm{eV}$ for the crystal relative to $\mathrm{C}_{2} \mathrm{H}_{2}$ in solid $\mathrm{Ne}$ (Fig. 22). (b) An intense crystal transition originating at $8.2 \mathrm{eV}$, which shows the following characteristics. (b1) A short vibrational progression of $1300 \mathrm{~cm}^{-1}$, as is the case for the Rydberg state of acetylene. (b2) A large spectral shift $(0.75 \mathrm{eV})$ in the crystal relative to acetylene in $\mathrm{Ne}$ (Fig. 22). This transition is assigned to the $n=1$ intermediate Wannier excitation in crystalline acetylene, whose parentage can be traced to the first Rydberg state of the acetylene molecule. 
A novel experimental approach to the study of molecular Rydberg states in a dense medium will originate from spectroscopic studies of van der Waals (vdW) molecules ${ }^{60}$ and clusters. ${ }^{61}$ Let us consider a vdW molecule $M \cdot R$, consisting of a diatomic or polyatomic molecule, $M$, bound to a rare-gas atom, $R$, e.g., N0.Ar ${ }^{62}$ or benzene-Ar. A Rydberg excitation of $M$ will exert short-range $M-R$ repulsive interaction, whereupon the potential curve will be repulsive, resulting in a dissociative continuum and manifesting direct $M-R$ photodissociation. Increasing the size of the vdW complex by adding more $R$ atoms will result in a $M_{n}$ large cluster. The Rydberg states of $M$ in a large cluster will not lead to direct dissociation but rather to nuclear relaxation around the excited state, resulting in the expansion of the cluster around the extravalence molecular excitations. The spectroscopy of Rydberg states of vdW molecules and clusters will provide direct information on medium effects on molecular Rydberg excitations, as explored from the microscopic point of view.

\section{EXCITED-STATE DYNAMICS IN SOLID RARE GASES}

Up to this point we have been concerned with the electronic structure of pure and doped insulators, which provide the essential input data for the elucidation of excited-state dynamics. Two general classes of relaxation processes in electronically excited states can be considered.

A. Intrastate processes. The system is produced in a nonequilibrium nuclear configuration, so that the balance of interatomic (or intramolecular) interactions is destroyed. Nuclear relaxation occurs within a single electronically excited state. The following phenomena correspond to this category: 
(A1) Exciton trapping by nuclear deformation in pure RGS and in rare-gas liquids. $1,22,63-67$

(A2) Lattice relaxation around excited impurity states in RGS. ${ }^{68-70}$

(A3) "Bubble" formation around electronically excited states in Tiquids.'

(A4) Medium-induced vibrational relaxation of $R_{2}$ * electronicallyvibrationally excited diatomic molecules in RGS and in liquid rare gases. 22

B. Interstate processes. The relaxation process is accompanied by a change in the electronic states, involving partial or complete interconversion of electronic energy into phonons. Typical examples are:

(B1) Electron-hole recombination. 6

(B2) Electronic relaxation of exciton s.tates in RGS. 22

(B3) Electronic relaxation of impurity states in RGS and in liquid rare gases. $22,68-70$

(B4) Electronic energy transfer by "free" excitons in RGS. 22,57,71-74

(B5) Electronic energy transfer between localized states in solid and in liquid insulators. $1,22,75-77$ 
We shall now proceed to discuss several examples for intrastate and interstate relaxation processes, emphasizing the role of molecular energetics and relaxation in condensed-phase dynamics.

\section{SELF-TRAPPING OF EXCITONS IN SOLID RARE GASES}

The emission spectra of RGS, ${ }^{63-67}$ summarized in Fig. 23, provide interesting information concerning exciton trapping by nuclear deformation. The prominent emission bands from pure solid $\mathrm{Ar}, \mathrm{Kr}$ and $\mathrm{Xe}$ are characterized by the following features:

(1) Broad luminescence bands are observed, exhibiting large (1-2 eV) Stokes shifts relative to the lowest $n=1\left({ }^{2} P_{3 / 2}\right)$ excitonic absorption.

(2) The lineshapes of these emission bands are nearly Gaussian.

(3) Similar emission spectra are exhibited in the solid, in the liquid and in the cold gas.

These 1 uminescence bands are due to ${ }^{63}$ the emission from the electronically excited, vibrationally relaxed, homonuclear rare-gas molecule, ${ }^{78} \mathrm{R}_{2}{ }^{*}$. From the schematic potential surfaces of diatomic rare-gas molecules, portrayed in Fig. 24, we note that the ground state is characterized by a shallow van der Waals potential. In the lowest excited ${ }^{3} \sum_{u}$ and ${ }^{1} \sum_{u}$ states the binding energy is high $(\sim 1-2 \mathrm{eV})$. 
Electronic excitation results in a considerable decrease in the equilibrium separation of $R_{2}$, which is reduced by about $30 \%$ relative to the ground state. Emission from the ground vibrational level of $R_{2}$ * will result in $R_{2}$ in its strongly repulsive part of the potential, so that the two $R$ atoms will fly apart. The large Stokes shift in emission and the broad, Gaussian, emission lineshapes in solid $\mathrm{Ar}, \mathrm{Kr}$ and $\mathrm{Xe}$ can readily be rationalized on the basis of this molecular picture. Furthermore, the practical invariance of the emission spectra with respect to the state of aggregation of the rare gas is also consistent with this molecular description of the emitting center in these RGS.

The emission of solid Ne drastically differs from the emission spectra of the heavier RGS. The prominent emission band of solid $\mathrm{Ne}$ is relatively narrow, peaking at $16.7 \mathrm{eV}$ (Fig. 23), which is Stokes shifted by $0.7 \mathrm{eV}$ to lower energies relative to the $n=1\left({ }^{2} \mathrm{P}_{3 / 2}\right)$ exciton states. 66,67 This emission originates from a medium-relaxed atomic state of $\mathrm{Ne} .66,67$ Medium relaxation results in dilation around the electronic excitation, so that perturbations on the atomic excitation are minor. Indeed, the energy of the prominent emission band in solid Ne is close to the low atomic excitations. In some experiments a splitting of this "atomic type" emission into three components was observed, which was correlated with the 
${ }^{1} P_{1},{ }^{3} P_{1}$ and ${ }^{3} P_{0}$ states. In addition to the prominent emission band, solid Ne exhibits another broad emission band located towards lower energies, which is assigned to the decay of the vibrationally unrelaxed $\mathrm{Ne}_{2}{ }^{*}$ diatomic molecule.

To summarize the phenomenology of the emission spectroscopy of RGS, we would like to point out that four distinct emission bands were observed, which will now be listed in the order of increasing energy.

(a) Emission from a free exciton state. The quantum yield for this emission

$$
Y=\tau_{t} / \tau_{R}
$$

is given in terms of the ratio between the exciton trapping time, $\tau_{t}$, and the pure radiative lifetime $\tau_{R}$ of the free exciton. For pure solid Xe, the experimental data indicate that ${ }^{65} \mathrm{\sim} \sim 10^{-3}$. As $\tau_{R} \simeq 10^{-9} \mathrm{sec}$, we estimate $\tau_{t} \simeq 10^{-12} \mathrm{sec}$, indicating the occurrence of efficient exciton trapping on the psec timescale.

(b) Emission from a localized atomic excitation. This relatively narrow emission band is somewhat red shifted relative to the exciton state, indicating the reduction of repulsive matrix interactions due to lattice dilation. This emission band is prominent in solid $\mathrm{Ne}$.

(c) Emission from a vibrationally hot diatomic $R_{2}$ * molecule. In this case exciton trapping via the formation of $R_{2}$ * took place; however, vibrational relaxation is slow on the timescale of the radiative decay of the diatomic molecule. The secondary emission band in solid $\mathrm{Ne}$ is due to the emission. 
(d) Emission from vibrationally relaxed $R_{2}$ * excited molecules. The prominent emission bands of solid $\mathrm{Ar}, \mathrm{Kr}$ and $\mathrm{Ne}$ correspond to this category.

In the language of solid-state physics, the emission from a $R_{2}$ * center corresponds to two-center trapping, while the emission from an atomic state is attributed to one-center trapping. Two mechanisms for exciton two-center and one-center trapping in RGS were advanced.

A. The molecular approach. ${ }^{22}$ Two-center localization in solid Ar, $\mathrm{Kr}$ and $\mathrm{Xe}$ originates from the formation of $\mathrm{R}_{2}$ * at high vibrational states, followed by efficient vibrational relaxation of the diatomic molecule. In view of the low vibrational frequencies of $\mathrm{Ar}_{2}{ }^{*}, \mathrm{Kr}_{2}{ }^{*}$ and $\mathrm{Xe}_{2}{ }^{*}$, which are comparable to the Debye frequency $\hbar_{\omega_{0}}$ of the solid, vibrational relaxation is very efficient on the timescale of their radiative decay. In solid $\mathrm{Ne}$ vibrational relaxation is slow due to the high vibrational frequency of $\mathrm{Ne}_{2}$ * relative to the Debye frequency. One can then consider a local picture for exciton trapping, whose assets are:

(A1) Predominance of the molecular point of view. The description of exciton trapping is universal for solids, liquids and gases.

(A2) The weak $\left(Y \sim 10^{-3}\right)$ free-exciton emission from solid $\mathrm{Xe}$ can be rationalized in terms of a low branching ratio from excited states which evaded vibrational relaxation. 
(A3) The absence of relaxed molecular emission from solid $\mathrm{Ne}$ is explained.

From the point of view of general methodology, the molecular model disregards excitonic and solid-state effects, which are emphasized by the alternative approach to the problem.

B. The solid-state continuum mode1. 79,80 Exciton localization in RGS is of considerable methodological interest, as in these solids shortrange coupling with acoustic phonons prevails, while long-range coupling to polar modes does not exist. Toyozawa ${ }^{79}$ and Rashba ${ }^{80}$ considered the transition from free to localized state of the exciton, which occurs when the lattice relaxation energy, $E_{L R}$, is comparable to the exciton bandwidth b. The localization process due to lattice distortion can be visualized as the splitting of a bound state from the exciton band. When $E_{L R}$ is sufficiently large, the localized state is characterized by a minimum on the adiabatic potential surface, which is separated from the free exciton state by a potential barrier $\mathrm{H}_{\mathrm{m}}$. On the basis of an isotropic continuum, where the lattice distortion corresponds to an elastic dilation, the barrier height is given by $H_{m}=4 b^{3} / 27 E_{L R}{ }^{2}$. According to the continuum model, two conditions have to be satisfied for the observation of selftrapping in a solid. Firstly, the energetic criterion $E_{L R} \simeq b$ has to be satisfied. Secondly, a dynamic criterion $H_{m} \leqslant \hbar \omega_{D}$ has to be obeyed to ensure 
nuclear tunnelling from the free exciton to the selftrapped state. A schematic energetic scheme appropriate for selftrapping is portrayed in Fig. 25. The continuum model is not specific concerning the microscopic aspects of selftrapping. Two stable configurations of the localized state are of particular interest, which involve (Fig. 26): (1) The onecenter, atomic, trapped exciton. Here, the nearest neighbors displace symmetrically around the excitation. (2) The two-center trapped excitations. Here, the two neighboring atoms displace axially. The relative energetic stability of the one-center and the two-center excitation can be assessed, in principle, on the basis of an excitonic mode $1^{81}$ (Fig. 26), which considers the change in the diagonal site energies $U^{\prime}$ and the change in the off-diagonal energies $V^{\prime}$ accompanying lattice displacements. Large diagonal deformations result in one-center trapping, while large off-diagonal deformations give twocenter trapping. The continuum model modified to incorporate the microscopic nature of exciton trapping (Fig. 26) in RGS led to the following predictions.

(B1) The two-center trapped exciton $R_{2}{ }^{*}$ is energetically favored over the free exciton for all RGS.

(B2) The two-center trapped exciton is energetically favored over the one-center trapped state for all RGS. This prediction is invariance with the experimental data for solid $\mathrm{Ne}$.

(B3) In solid $\mathrm{Ne}$ and $\mathrm{Ar}$, the barrier height $H_{m}$ is low relative to $h_{\omega_{D}}$ and very efficient trapping occurs. 
(B4) In solid $\mathrm{Xe}$, the barrier height for two-center localization is large, so that the free state should be observed. The low $\left(Y \sim 10^{-3}\right)$ quantum yield for emission from free excitons in solid $\mathrm{Xe}$ is blamed on trapping and imperfections.

It appears that at present it is rather difficult to assess the relative merits of the molecular and the solid-state models on the basis of the confrontation of predictions with the experimental facts. The molecular model has a slight edge, as it correctly predicts one-center localization in solid $\mathrm{Ne}$, indicating a slight superiority of chemical concepts over the physical model.

Further support for the "chemical" description of selftrapped excitons in RGS stems from recent experimental information concerning transient absorption studies ${ }^{82}$ and time-resolved studies. 83 Transient absorption studies $^{82}$ of the selftrapped exciton in solid $\mathrm{Ar}$ and $\mathrm{Kr}$ have established that $R_{2}$ * corresponds to the ${ }^{3} \sum_{u}$ state of the homonuclear rare-gas diatomic molecule. These studies provide further support for the molecular descri.ption of the selftrapped exciton in heavy RGS. Time-resolved studies of the emission from solid $\mathrm{Ar}, \mathrm{Kr}$ and $\mathrm{Xe}$ (Figs. 27 and 28) have established the occurrence of multiple decay. In solid Xe (Fig. 27) the decay is characterized by both a short (nsec) and a long (nsec) temperaturedependent component. Furthermore, the spectral distribution of the short 
and long components is different (Fig. 28). The short component was tentatively attributed to the radiative decay of the ${ }^{1} \Sigma^{-}$molecular

state, while the long component(s) are due to the decay of the ${ }^{3} \sum^{-}$ molecular state, which may be subjected to crystal field splitting. These time-resolved data again demonstrate the prominence of the molecular point of view for the description of the selftrapped exciton in heavy RGS. It should, however, be emphasized that both the transient absorption and the time-resolved studies interrogate the selftrapped exciton on a "long" (nsec and longer) timescale and do not provide information on the trapping process which occurs on the psec timescale. The molecular and the solid-state models for exciton trapping should not be considered as orthogonal, but rather complementary and supplementary, so that the synthesis of chemical and physical concepts will provide a general picture of the dynamics of exciton localization in simple insulators.

\section{LATTICE RELAXATION AROUND IMPURITY STATES}

The one-center localization of the exciton in pure solid $\mathrm{Ne}$ involves symmetric radial displacement of the neighboring atoms away from the electronically excited state. An analogous phenomenon is ubiquitous for electronically excited atomic impurity states in RGS. In these systems 
short-range repulsive interactions exerted by the excited atom on its neighbors result in an appreciable increase of the average separation between the electronically excited atom on its nearest host atoms. The electronically-vibrationally excited state, populated by a vertical transition (Fig. 29) will relax subsequently by phonon emission to a new equilibrium configuration. ${ }^{6}$ The configurational diagrams for the ground state and electronically excited state of an impurity in a RGS, as portrayed in Fig. 2y, correspond to the strong electron-phonon coupling limit, which is characterized by a large horizontal displacement of the minima of the potential surfaces. ${ }^{6}$ The absorption lineshape is expected to be Gaussian with a linewidth ${ }^{6}$

$$
H=H_{0}\left(\operatorname{coth} \frac{\hbar\langle\omega\rangle}{2 k T}\right)^{1 / 2},
$$

where $\langle\omega\rangle$ is a characteristic phonon frequency. These expectations are borne out by the absorption spectra of Xe impurity in a $1 \mathrm{~cm}$ thick $\mathrm{Ar}$ crystal $^{84}$ (Figs. 30 and 31 ). The Gaussian lineshape and the temperature dependence of the linewidth clearly demonstrate that the system corresponds to the strong coupling situation. The large lattice relaxation around impurity states has a profound effect on the energetics of the emission from impurity states. The large configurational change around the impurity center will drastically reduce the short-range repulsive interactions, which in turn have contributed to a large blue spectral shift in absorption 
relative to the atomic excitation. Accordingly, one expects a large Stokes shift between absorption and emission. The older experimental data $^{68,69}$ for the emission of Xe in solid and in liquid Ar (Fig. 32) excited by $X$-rays and the newer experimental data ${ }^{70}$ for the emission of Ar, $\mathrm{Kr}$ and $\mathrm{Xe}$ in solid $\mathrm{Ne}$ excited by synchrotron radiation (Table IX) demonstrate the existence of such a large Stokes shift. Furthemore, the large symmetric lattice dilation around the impurity state results in appreciable reduction of the impurity medium interaction, so that the spectral shift of the emission relative to the atomic excitation is small (Table IX). To provide a physically transparent demonstration of the effect of medium dilation around the excited impurity state, we present in Fig. 33 the excitation (absorption) spectrum and the emission spectrum of $\mathrm{Xe}$ in $\mathrm{Ne} .^{70}$ The absorption and the emission bands for the $6 s[3 / 2], 6 s[1 / 2]$ and $7 s[3 / 2]$ atomic states are connected by a Messing plot (see section $V$ ) of the energies versus the density and are plotted in Fig. 33 to give a linear scale for the nearest-neighbor separation. 1,70 The emission spectra correspond to medium perturbations exerted at an average Xe-Ne separation of $\sim 4.5 \AA$, which exceeds by $30 \%$ the ground state Xe-Ne spacing of $3.1 \AA$. Figure 33 demonstrates nicely the formation of a "microscopic cavity"7,68 around the electronically excited impurity state. 


\section{$X$. ELECTRONIC RELAXATION OF IMPURITY STATES}

Figure 33 clearly demonstrates that prominent emission of $\mathrm{Xe}$ impurity in solid Ne occurs from the three excited states; $6 s[3 / 2]$, $6 s[1 / 2]$ and $7 s[3 / 2]$. This observation is interesting and quite exceptional. In a variety of molecular excited states 85 and in impurity centers in solids, ${ }^{6}$ effective interstate electronic relaxation between highly excited states results in efficient nonradiative decay, rendering these high states to be ineffective in emission, so that emission is observed only from the lowest electronically excited state. The $\mathrm{Xe} / \mathrm{Ne}$ system, whose interstate dynamics is summarized in Fig. 34, as well as other atomic impurity states in RGS, provide examples for rather unique inefficient electronic relaxation between highly excited states, which is slow on the timescale of the radiative decay: ${ }^{70}$ The cardinal question is: Which states do emit? This question can be answered by invoking the energy gap law for electronic relaxation. ${ }^{85}$ The nonradiative rate, $w$, can be expressed in the form

$$
W=\frac{A}{(2 \pi \Delta E / h\langle\omega\rangle)^{T / 2}} \exp (-G) \exp (-\gamma \Delta E / h<\omega>)
$$

where $A\left(\simeq 10^{12}-10^{14} \mathrm{sec}^{-1}\right)$ is a pre-exponential factor, $G$ is the strength of electron-phonon coupling, $\gamma$ is a numerical parameter of the order of 
unity, $\langle\omega\rangle$ is a characteristic phonon frequency and $\Delta E$ is the electronic energy gap. The exponential decrease of $W$ with increasing $\Delta E$ makes electronic relaxation ineffective for large energy gaps. This expectation is in accord with the experimental data of Fig. 34, where large energy gaps prohibit electronic relaxation between high excited states.

\section{ELECTRONIC ENERGY TRANSFER IN SOLID RARE GASES}

Studies of electronic energy transfer in pure RGS are of interest to establish the features of exciton migration on an ultrashort timescale prior to exciton trapping. We have shown in section VIII that the lifetimes of "free" excitons are $\tau \sim 10^{-12}$ sec. These psec energy transfer phenomena are expected to provide interesting information on the nature of exciton dynamics, which may correspond to one of the following categories:

(a) Coherent exciton transport. The exciton lattice scattering is weak, so that the exciton mean free path $\Lambda$ considerably exceeds the lattice spacing $r_{c}$. The exciton dynamics is essentially determined by its group velocity, so that the displacement of the exciton at time $t$ is proportional to $t$.

(b) Incoherent exciton transport. When the exciton scattering by phonons, disorder, etc., is strong, the exciton mean free path is comparable to the lattice spacing, $\Lambda \approx r_{c}$. Exciton transport is now characterized by a 
diffusion coefficient $D$, while the diffusion length, $\ell$, is given by

$$
\ell=\left(D_{\tau}\right)^{1 / 2},
$$

where $\tau\left(\sim 10^{-12} \mathrm{sec}\right)$ is the "free" exciton lifetime.

To explore electronic energy transfer by "free" excitons, one has to utilize sensitive methods to interrogate dynamic processes on the psec timescale. Photoemission and fluorescence measurements provide adequate tools. Three methods were advanced for the study of the diffusion lengths of "free" excitons:

A. Exciton induced impurity ionization. $57,58,80$ For dilute impurity states intrinsic photoemission occurs ensuing from excitation of the host exciton states, which in turn transfers energy to the impurity, resulting in Penning-type ionization of the impurity (Fig. 35). Only "free" excitons can induce this ionization process, as the energy of $R_{2}$ * trapped excitons is too low, so that trapped excitons are inactive. The interrogation of impurity ionization by "free" excitons is conducted by the measurements of the photoenission yield curves or electron energy distribution curves at several photon energies. Figure 36 shows a typical exariple for the emission of benzene in $\mathrm{Xe}$, which originates from this mechanism. From the dependence of the photoemission yield on the excitation wavelength and impurity, the exciton diffusion length is obtained.

B. Extrinsic electronic emission via energy transfer to a metal substrate. $71-73$ This is a "classical" energy transfer experiment via excitons (Fig. 37). Excitons are excited in a RGS below the direct photoemission 
threshold. These excitons diffuse to a metal substrate, which is used as an electrode, resulting in photoemission from the electrode (Fig. 37). By monitoring the dependence of the photo yield on the film thickness (Fig. 38) and the wavelength (Fig. 39), the exciton diffusion length can be inferred.

C. Energy transfer to contaminated boundary surface of a rare-gas film. $74,76,77$ This process reduces the quantum yield for luminescence. These luminescence quenching experiments provide information on the exciton diffusion length.

In Table $X$ we have summarized experimental data for the diffusion of "free" excitons in RGS. From these results it is apparent that:

(C1) The diffusion lengths considerably exceed the lattice constant.

(C2) The diffusion length in solid $\mathrm{Ne}$ is considerably higher than in the heavier RGS, presumably reflecting a longer lifetime for the free exciton, which is subjected to one-center localization in solid $\mathrm{Ne}$.

(C3) The diffusion coefficients of free excitons in $\mathrm{Ar}, \mathrm{Kr}$ and $\mathrm{Ne}$ are $D \sim 1 \mathrm{~cm}^{2} \mathrm{sec}^{-1}$. Such diffusion coefficients correspond to incoherent diffusive motion.

(C4) In RGS studies up to date, strong scattering of excitons by phonons and by crystal imperfections dominate the exciton dynamics. 


\section{I PERSPECTIVES}

The present overview of the energetics and dynamics of electronic excitations in large-gap insulators demonstrates the interplay between molecular and solid-state concepts. Solid-state theoretical concepts were invoked for the description of electronic excitations of pure solids and of impurity states. Nevertheless, the tight binding approach, which rests on the "molecular" properties of the constituents, provides a useful starting point. Furthermore, the quantum defect method provides a bridge between high solid-state excitation and the highly excited atomic (or molecular) states of the constituents. The molecular approach was also extremely useful for the elucidation of some intrastate relaxation phenomena, e.g., the nature of selftrapped excitons in pure materials, as well as the energetics of emission from excited impurity states. Last but not least, the molecular local approach is extremely relevant for the understanding of the energetics and dynamics in structurally disordered insulators, i.e., fluids and liquids. The merging between the molecular and solid-state points of view is of considerable interest. While our current understanding of the electron structure of the ground and excited states of insulators, such as RGS, is fairly complete, further information will be required for the elucidation of excited-state dynamics. Most of the experiments we would like to suggest are by no means easy. However, they appear to us to be worthile performing, and we suggest the following methods : 
(1) Determination of the homogeneous linewidths for excitons and impurity states by "hole burning" and laser fluorescence line narrowing in the vacuum UV.

(2) Probing of the retention of phase memory by the interrogation of coherent optical effects, i.e., free-induction decay or photon echoes in the vacuum UV.

(3) Studies of exciton diffusion in "good" well-annealed crystals of insulators to eliminate the effects of structural disorder effects.

(4) Studies of electronic energy transfer of high-energy excitations in an impurity band of binary large-gap insulators. Some novel information regarding the problem of Anderson localization 87,88 and dispersive transport $^{89}$ of excitons will emerge from such studies. 


\section{TABLE I}

Radii of the $a=1$ excitons for Ne, Ar, $\mathrm{Kr}$ and Xe in comparison with the nearest neighbor distances $r_{c}$ in a fcc-lattice

\begin{tabular}{l|l|l|l|l|} 
& $\mathrm{Ne}$ & $\mathrm{Ar}$ & $\mathrm{Kr}$ & $\mathrm{Xe}$ \\
\hline $\left.\mathrm{r}^{\mathrm{n}=1}\{\AA\}\right\}^{(a)}$ & & 1.8 & 2.5 & 3.2 \\
$\left.r_{c}\{\AA\}\right\}^{(b)}$ & 3.156 & 3.755 & 3.992 & 4.335
\end{tabular}

(a) Calculated with equation (3.3).

(b) G. K. Horton, Am. J. Phys. 36, 93 (1968). 
TABLE II Energetic Parameters for Exciton States in Pure RGS

$\begin{array}{lcccc}\text { Solid } & E_{G} & B & \Delta E_{S O} \\ (\mathrm{eV}) & (\mathrm{eV}) & 0.09 & \Delta \\ \mathrm{Ne} & 21.58 & 4.98 & 0.18 & 0.86 \\ \mathrm{Ar} & 14.16 & 2.36 & 0.69 & 0.26 \\ \mathrm{Kr} & 11.61 & 1.53 & 1.32 & 0.09 \\ \mathrm{Xe} & 9.33 & 1.02 & & 0.06\end{array}$




\section{Table III}

Comparison between experimental transition energies for bulk excitons in solid neon and other parameters with theoretical calculations based on fits by the QDM. $B_{1}=E_{G}-E(n=1)$ are the experimental binding energies of the $n=1$-excitons and $\mu$ is the effective mass. All energies are in $\mathrm{eV}$.

\begin{tabular}{|c|c|c|c|c|c|c|}
\hline \multirow{4}{*}{$\begin{array}{c}\mathrm{J} \\
\mathrm{n}=1\end{array}$} & \multicolumn{2}{|c|}{ Experiment } & \multicolumn{2}{|c|}{ Theory } & \multirow{2}{*}{\multicolumn{2}{|c|}{ Ref. 34}} \\
\hline & Ref. 2 & (a) & \multicolumn{2}{|c|}{ Ref. 36} & & \\
\hline & $3 / 2$ & $1 / 2$ & (b) & & \multicolumn{2}{|c|}{ (c) } \\
\hline & 17.36 & 17.50 & 17.75 & 17.85 & 17.58 & 17.79 \\
\hline 2 & 20.25 & 20.36 & 20.32 & 20.42 & 20.24 & 20.35 \\
\hline 3 & 20.94 & 21.02 & 20.94 & 21.04 & 20.91 & 21.03 \\
\hline 4 & 21.19 & 21.29 & 21.19 & 21.29 & 21.19 & 21.31 \\
\hline 5 & 21.32 & & 21.31 & 21.41 & 21.33 & 21.45 \\
\hline $\mathrm{E}_{\mathrm{G}}$ & 21.58 & 21.62 & 21.55 & 21.65 & 21.61 & 21.73 \\
\hline${ }^{B}{ }_{1}$ & 4.22 & 4.12 & & & & \\
\hline$\Delta_{\text {so }}$ & & & & & & .12 \\
\hline$\delta$ & 0.28 & 0.24 & 0.35 & 0.35 & 20.5 & 20.5 \\
\hline$\mu$ & 0.8 & 0.7 & & & & .97 \\
\hline
\end{tabular}

(a) V. Saile, and E.E. Koch, Phys. Rev. B20, 784 (1979) Ref. 27

(b) R. Resta, phys. stat. sol.(b) $\underline{86}, 627$ (1978), Ref. 36; gap energies are obtained by a fit of the experimental data Ref. 27, and $\Delta_{\text {so }}$ is the atomic value. From these gap energies we subtracted the theoretical exciton binding energies. The values for $\delta$ vary only little with $\mathrm{n}$ for the theoretical QDM results. For more details see Ref. 27.

(c) L. Resca, R. Resta and S. Rodriguez, Sol. State. Comm. 26, 849 (1978), and Phys. Rev. B18, 696 (1978), Ref. 34 
TABLE IV Longitudinal Transverse Splittings for Bulk

$$
\text { Excitons in RGS(a) (in eV) }
$$

Ne $\mathrm{Ar} \quad \mathrm{Kr} \quad \mathrm{Xe}$

\begin{tabular}{|c|c|c|c|c|c|c|c|c|}
\hline$n=1$ & $3 / 2$ & $1 / 2$ & $3 / 2$ & $1 / 2$ & $3 / 2$ & $1 / 2$ & $3 / 2$ & $1 / 2$ \\
\hline$\Delta_{\text {LO-TO }}^{\text {opt. }}$ & - & 0.27 & - & 0.26 & 0.12 & 0.09 & 0.06 & - \\
\hline
\end{tabular}

(a) V. Saile, and E.E. Koch, Phys. Rev. B20, 784 (1979), and unpublished results. 


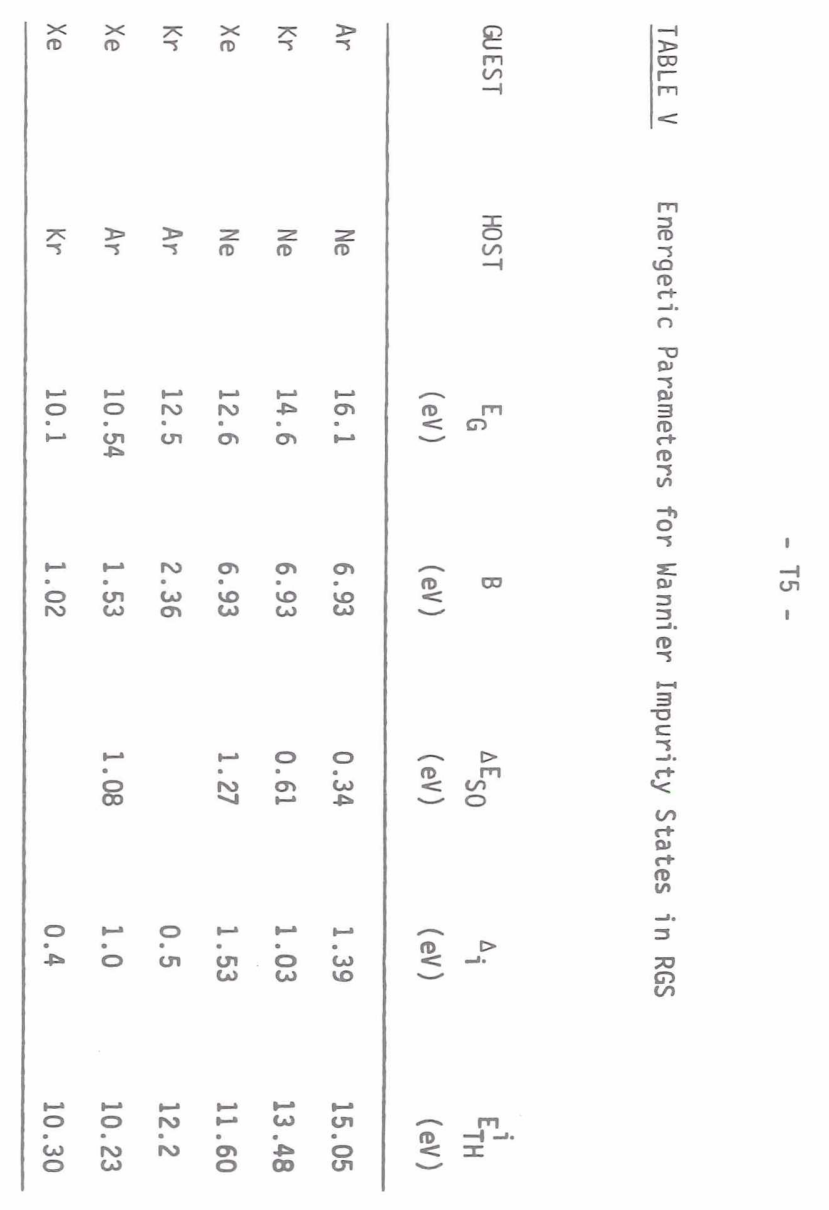

\section{TABLE VI}

Experiment $V_{0}$ scale for solid and liquid rare gases obtaines from optical and photoemission experiments (see table 3 ) compared to the calculation of $\mathrm{V}_{0}$ based on the SPRINGETT, JORTNER, COHEN (SJC) (1968) therory. A superscript $\mathrm{i}$ denotes values for the impurity states. $V_{O}=E_{G}-E_{T I I}=U_{p}+T$. $U_{p}$ : background polarisation potential; T: kinetic energy term. $a, b$

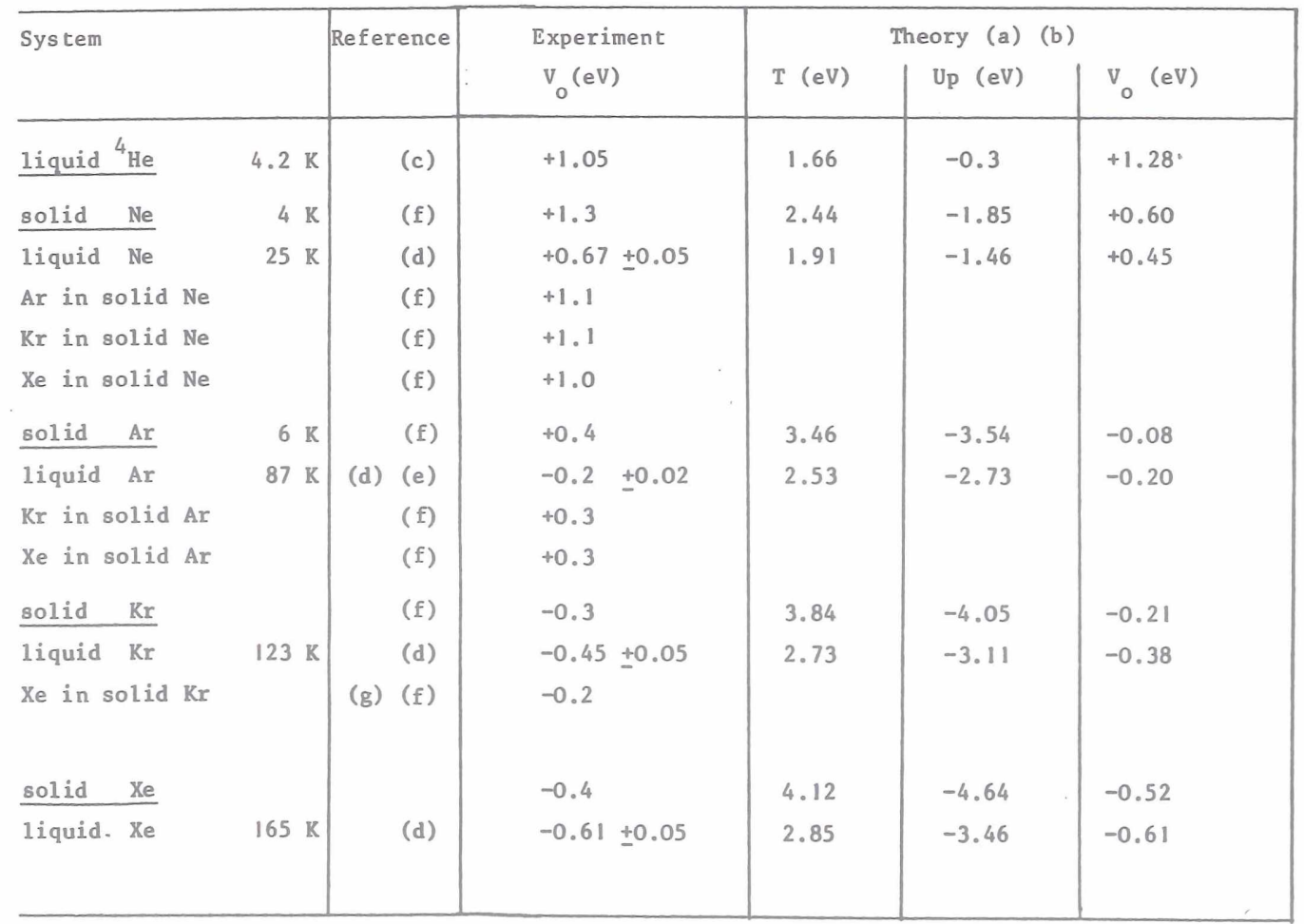


Reference for Table VI

(a) B.E. Springett, M.H. Cohen and J. Jortner,

J. Chem. Phys. 48, 2720 (1968).

(b) J. Jortner and A. Gaathon, Canad.J.Chem. 55, 1801 (1977).

(c) W.I. Sommer, Phys.Rev. Letter 12, 271 (1964).

(d) W.K. Tauchert, Ph.D. Thesis, Freie Universitat Berlin, Berlin, W. Germany, 1975, and

W.K. Tauchert and W.F. Schmidt, Z.Naturforsch. 30a, 1085 (1975).

(e) J. Leckner el a1., Phys.Rev. 156, 351 (1972). 
TABLE VII Spectral Shift of the $n=1$ Transitions of Molecular Impurities in Solids Relative to the Electronic Origin of the Lowest Rydberg (in $\mathrm{cm}^{-1}$ )

\begin{tabular}{lcccccc} 
& & & & \\
MATRIX & $\mathrm{Xe}$ & $\mathrm{Kr}$ & $\mathrm{Ar}$ & $\mathrm{Ne}$ & $\mathrm{CF}_{4}$ & $\mathrm{~N}_{2}$ \\
\hline $\mathrm{CH}_{3} \mathrm{I}$ & $\cdots$ & 5095 & 6480 & 8950 & 12880 & $\ldots$ \\
$\mathrm{C}_{6} \mathrm{H}_{6}$ & 1400 & 2920 & 5640 & 6800 & $\ldots$ & 5520 \\
$\mathrm{C}_{2} \mathrm{H}_{4}$ & 2850 & 4160 & 5720 & 6330 & $\ldots$ & $\ldots$ \\
$\mathrm{C}_{2} \mathrm{H}_{2}$ & $\ldots$ & 4210 & 6650 & 6910 & 8700 & $\ldots$ \\
\hline
\end{tabular}




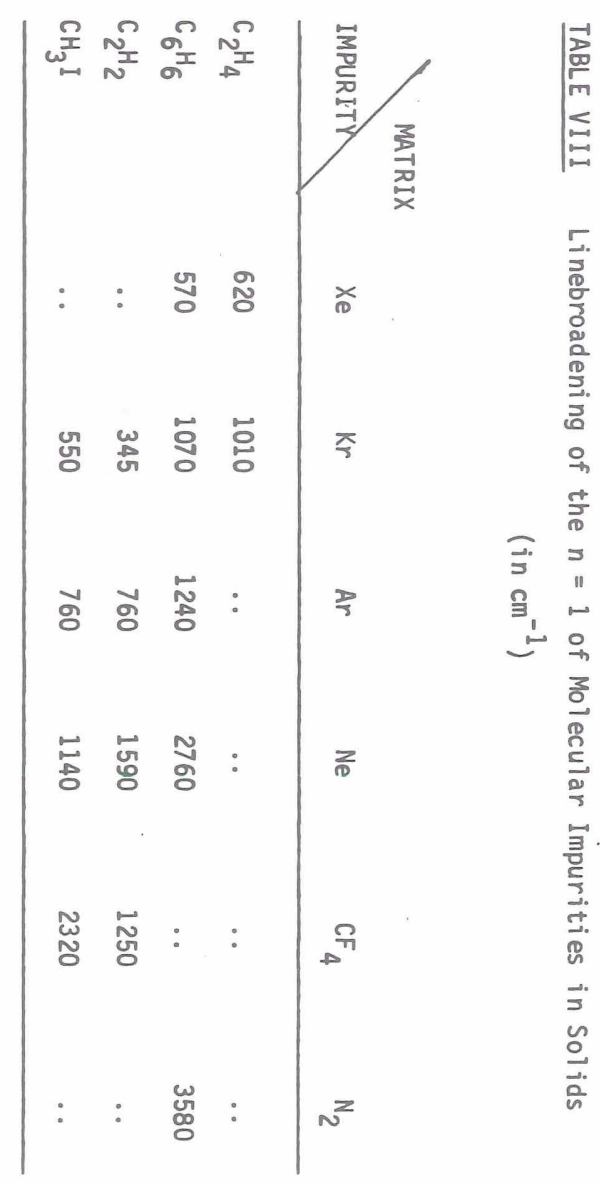

TABLE IX

Energies of en:ission bands (E) and comparison with the corresponding states in absorption (A) and in the free atom $(G)$. Energies in $\mathrm{eV}$.

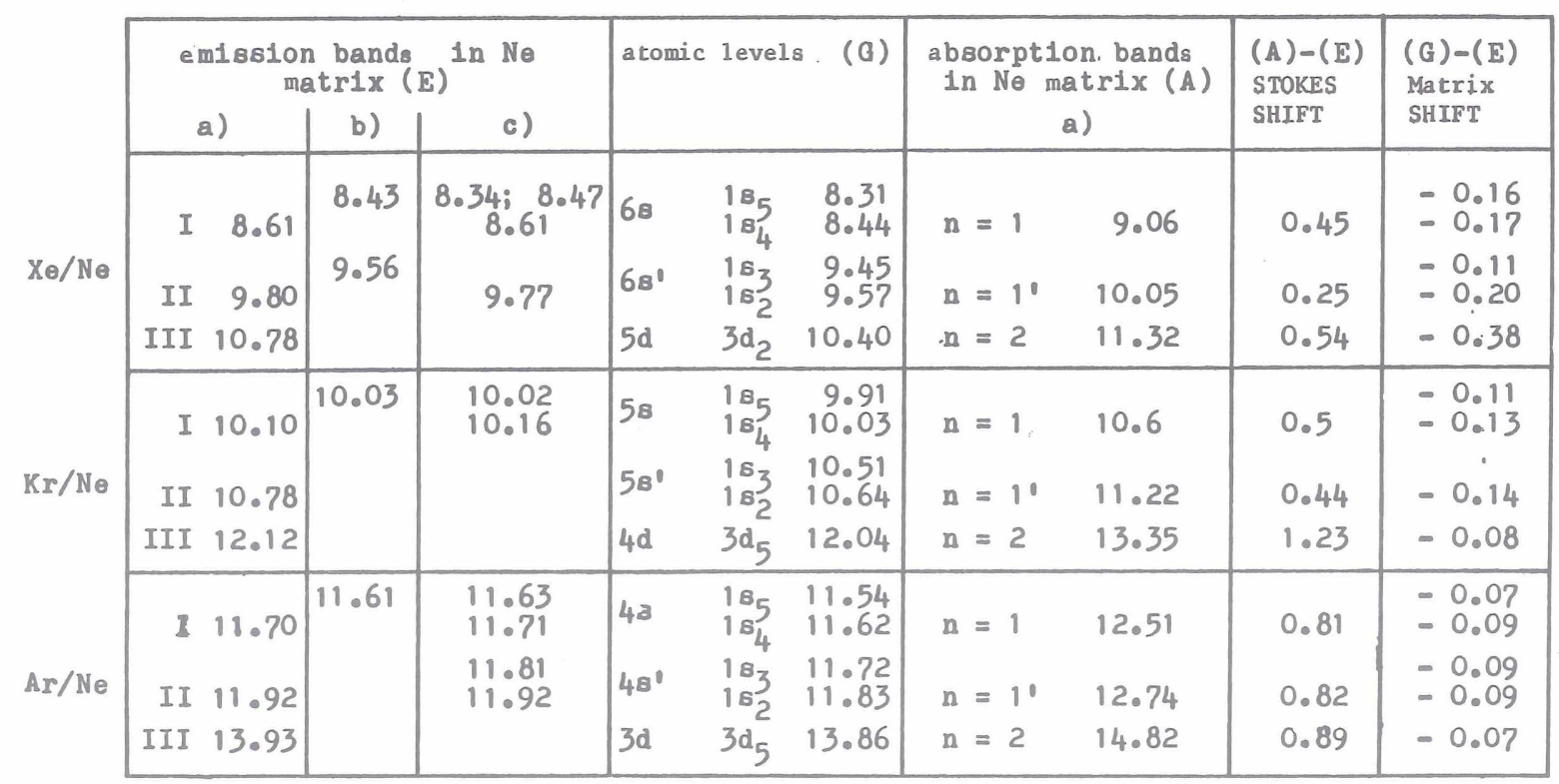

\footnotetext{
a) Hahn and Schwentner, (reference 70).

b) Gedanken, Raz and Jortner, (reference 69).

c) Schuberth and Creuzburg, Phys.Stat.Solidi B90, 169 (1978).
} 
TABLE X Diffusion Lengths for Energy Transfer to Guest Atoms and to Boundaries (in $\AA$ ).

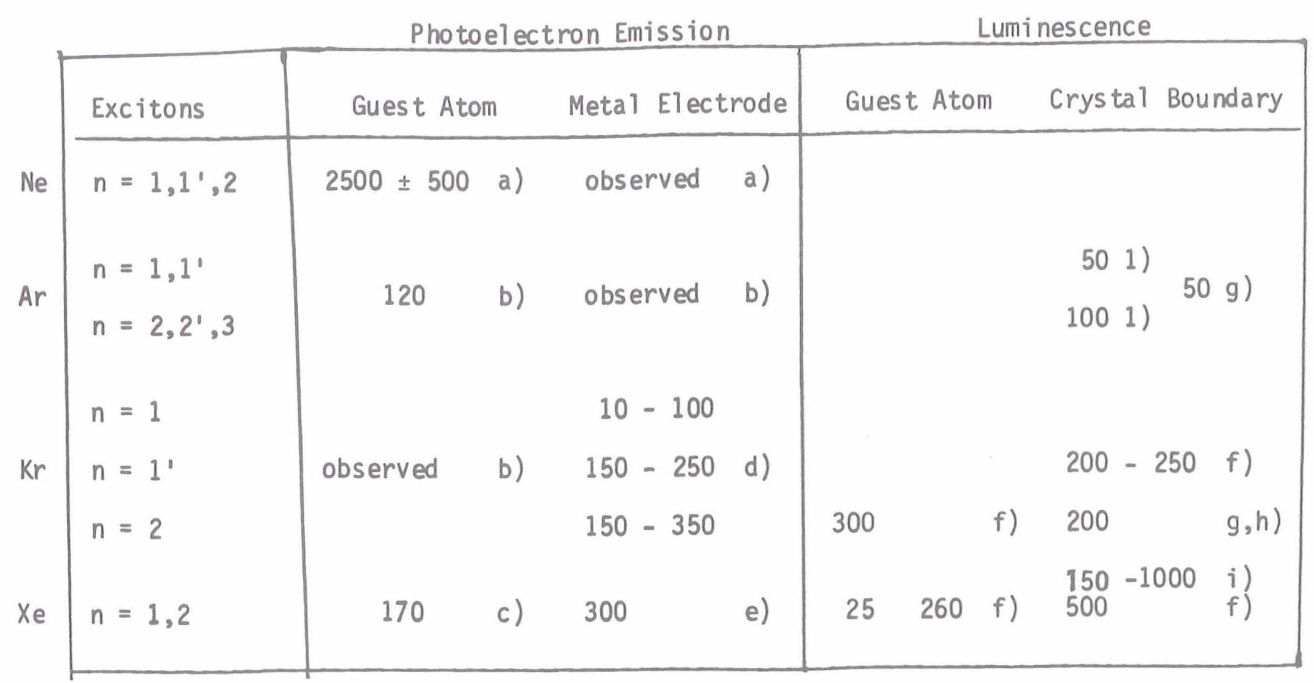

a) Pudewi11, Himpse1, Saile, Schwentner, Skibowski, Koch, and Jortner, J. Chem. Phys. $\underline{65}, 5226$ (1976).

b) Ophir, Raz, Jortner, Saile, Schwentner, Koch, Skibowski, and Steinmann, J. Chem. Phys. 62, 650 (1975).

c) Ophir, Raz, and Jortner, Phys. Rev. Lett, 39, 415 (1974).

d) Schwentner, Rudolf, and Martens, Phys. Stat. Solidi (1980).

e) Ophir, Schwentner, Raz, Skibowski, and Jortner, J. Chem. Phys. 63, 1075 (1975).

f) Ackermann, Thesis, Hamburg University (1976).

g) Ackermann, Brodmann, Tolkiehn, Zimmerer, Haensel, and Hahn, J. Lum. 12/13, 315 (1976).

h) Ackermann, Brodmann, Hahn, Suzuki, and Zimmerer, Phys. Stat. Solidi 74B, 579 (1976).

i) Gerick, Thesis, Hamburg University (1977). 

${ }^{1}$ N. Schwentner, E.E. Koch, and J.Jortner, Electronic Excitations in Condensed Rare Gases, Springer Tracts in Modern Physics, in press

2A.S. Davydov, Theory of Molecular Excitons (Plenum Press, New York, 1971).

${ }^{3}$ D.P. Craig, and S.H. Whalmsly, Excitons in Molecular Crystals. (Benjamin, New York, 1968).

${ }^{4}$ S.A. Rice, and J. Jortner, in Physics and Chemistry of the Organic Solid State, edited by F. Fox, M. Labes, and A. Weissberger (Interscience Publishers, New York, 1967), volume III, chapter 4.

${ }^{5}$ R.S. Knox, Theory of Excitons (Academic Press, New York, 1963).

${ }^{6}$. Kubo, and H. Kamimura, Dynamical Processes in Solid State Optics (Benjamin, New York, 1967).

7M. Brith, and 0. Schnepp, Mol. Phys. 9, 473 (1965).

${ }^{8}$ R. Schraber, and S.E. Webber, J. Chem. Phys. 52, 3977 (1971).

${ }^{9}$ E. Baursey, V. Chandrasekharan, P. Gürtler, E.E. Koch, P. Kunsch, and V. Saile, Phys. Rev. Lett. 41, 1516 (1978).

10P. Gürtler, and E.E. Koch, Chem. Phys. 49, 305 (1980).

${ }^{11}$ P. Gürtler, Ph.D. Thesis, Hamburg University (1979).

12M.H. Cohen, and F. Keffer, Phys. Rev. 99, 1128 (1955).

13W.R. Heller, and A. Marcus, Phys. Rev. 84, 809 (1951).

${ }^{14}$ A.S. Davydov, and E.N. Myasnikov, Phys. Stat. Solidi 20, 153 (1967). 
${ }^{15}$ E.I. Rashba, Sov. Phys.-JETP 27, 292 (1968).

16 J. Klafter, and J. Jortner, Chem. Phys. 47, 25 (1980).

${ }^{17}$ K. K. Rebane, Impurity Spectra of Solids (Plenum Press, New York, 1970).

${ }^{18}$ H.J. Lau, Diplom. Thesis, Hamburg University (1982).

Iy J.-H. Fock, H.J. Lau, and E.E. Koch, to be published

${ }^{20}$ C. Cossart-Magos, and S. Leach, J. Chern. Soc. Farad. Trans. 78, 1477 (1982) see also C. Friedh, L. Asbrink and E. Lindinolin, Cheri.Phys. Letters 27, 1609 (1978)

${ }^{21}$ S. Webber, S.A. Rice and J. Jortner, J. Chem.. Phys. 42, 1907 (1965).

22J. Jortner, in Vacuum U1traviolet Radiation Physics, edited by E.E. Koch, R. Haensel, and C. Kunz (Perganion Vieweg, 1974), p. 263.

${ }^{23}$ W. Kohn, Solid State Physics (Academic Press, New York, 1958), Vol. 5, p. 258.

${ }^{24} 0$. Schnepp, and K. Dressler, J. Chem. Phys. $\underline{33}, 49$ (1960).

${ }^{25}$ G. Baldini, Phys. Rev. 128, 1562 (1962).

26 B. Sonntag, in Rare Gas Solids, edited by M.K. Klein, and J.A. Venables (Academic Press, New York, 1977), Vo1. II, p. 1021.

${ }^{27}$ V. Saile, and E.E. Koch, Phys. Rev. B20, 784 (1979).

${ }^{28}$ V. Saile, and E.E. Koch, Phys. Rev. B21, 4892 (1980).

29. Hermanson, and J.C. Phillips, Phys. Rev. 150, 652 (1967).

30J. Hermanson, Phys. Rev. 150, 660 (1967).

${ }^{31}$ J.C. Phillips, Solid State Physics (Academic Press, New York, 1966), Vol. 18, p. 55. 
32W. Andneoni, M. Altarelli, and F. Bassani, Phys. Rev. B11, 2352 (1975).

33 W. Andreoni, W. Perrot, and F. Bassani, Phys. Rev. B14, 3589 (1976).

${ }^{34}$ L. Resca, R. Resta, and S. Rodriguez, Phys. Rev. B18, 696 (1978); B18, 702 (1978).

35L. Resca, and S. Rodriguez, Phys. Rev. B17, 3334 (1978).

${ }^{36}$ R. Resta, Phys. Stat. Solidi $\underline{86,} 627$ (1978).

${ }^{37}$ G. Baldini, and R.S. Knox, Phys. Rev. Lett. 11, 127 (1963).

${ }^{38}$ G. Baldini, Phys. Rev. 137A, 508 (1965).

${ }^{39}$ A. Gedanken, B. Raz, and J. Jortner, J. Chem. Phys. 58, 1178 (1973).

${ }^{40}$ D. Pudewill, F.J. Himpsel, V. Saile, N.Schwentner, M. Skibowski, and E.E. Koch, Phys. Stat. Solidi 74, 485 (1976).

${ }^{41}$ U. Asaf, and I.T. Steinberger, Phys. Rev. B10, 4464 (1974).

${ }^{42}$ B. Raz, and J. Jortner, Chem. Phys. Lett. 4, 511 (1970).

43. Jortner, and A. Gaathen, Cand. J. Chem. 55, 1801 (1977).

${ }^{44}$ B. Springett, J. Jortner, and M.H. Cohen, J. Chem. Phys. 48, 2720 (1968).

${ }^{45}$ N.F. Mott, and E.A. Davis, Electronic Processes in Non-Crystalline Solids (0xford University Press, 1971).

${ }^{46}$ S.A. Rice, and J. Jortner, J. Chem. Phys. 44, 4470 (1966).

${ }^{47}$ D. Beaglehole, Phys. Rev. Lett. 15, 551 (1965).

48 I.T. Steinberger, and U. Asaf, Phys. Rev. B8, 914 (1973). 
49. Raz, and J. Jortner, Proc. Prog. Soc. (London) A317, 113 (1970).

50 I. Messing, B. Raz, and J. Jortner, Chem. Phys. 23, 23 (1977).

51. Messing, B. Raz, J. Jortner, J. Chem. Phys. 66, 2239 (1977).

52I. Messing, B. Raz and J. Jortner, J. Chem. Phys. 66, 4577 (1977).

${ }^{53}$ U. Fano, Phys. Rev. 124, 1866 (1961).

${ }^{54}$ M. Robin, H. Basch, N.A. Kuebler, B.E. Kaplan, and J. Meinwald, J. Chem. Phys. $\underline{48}, 5037$ (1968).

55M. Robin, and N.A. Kuebler, J. Mol. Spect. $\underline{33}, 274$ (1970).

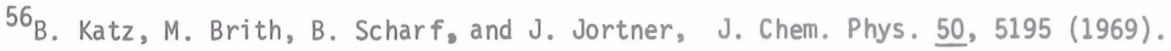

57 Z. Ophir, B. Raz, J. Jortner, V. Saile, N. Schwentner, E.E. Koch, M. Skibowski, and W. Steinmann, J. Chem. Phys. 62, 650 (1975).

${ }^{58}$ Z. Ophir, B. Raz, and J. Jortner, Phys. Rev. Lett. $\underline{33}, 415$ (1974).

${ }^{59}$ E.E. Koch, and J.H. Fock, unpublished results.

60D.H. Levy, Adv. Chem. Phys. $\underline{47}, 323$ (1981).

${ }^{61}$ A. Amirav, U. Even, and J. Jortner, J. Phys. Chem. (in press).

62P.R.R. Langridge-Smith, E. Carrasquillo, and D.H. Levy, J. Chem. Phys. 74, $6513(1981)$.

63J. Jortner, L. Meyer, S.A. Rice, and E.G. Wilson, J. Chem. Phys. 42, 4250 (1965).

${ }^{64}$ R. Hemüler, Thesis, University Ragensburg (1978). 
${ }^{65}$ F. Colletti, and A.M. Bonnot, Chem. Phys. Lett. $\underline{52}, 92$ (1978).

${ }^{66}$ R.E. Packard, F. Reif, and C.M. Surko, Phys. Rev. Lett. 25, 1435 (1970).

67 A. Gedanken, B. Raz, and J. Jortner, J. Chem. Phys. 53, 1630 (1973).

${ }^{68}$. Cheshnovsky, B. Raz, and J. Jortner, J. Chem. Phys. $\underline{57}, 4628$ (1972).

${ }^{69}$ A. Gedanken, B. Raz, and J. Jortner, J. Chem. Phys. 59, 5741 (1973).

70U. Hahn, and N. Schwentner, Chem. Phys. 48, 53 (1980); J. Lum. 18/19, 23 (1979).

${ }^{71}$ Z. Ophir, N. Schwentner, B. Raz, M. Skibowski, and J. Jortner, J. Chem. Phys. $\underline{63}, 1072$ (1975).

72. Pudewi11, F. Himpse1, V. Saile, M. Skibowski, N. Schwentner, E.E. Koch, and J. Jortner, J. Chem. Phys. 65, 5226 (1976).

${ }^{73}$ N. Schwentner, Applied Optics 19, 4104 (1980)

${ }^{74}$ Nagasawa and Namba, Optics Comm. 11, 152 (1974).

${ }^{75}$. Cheshnovsky, B. Raz, and J. Jortner, J. Chem. Phys. 59, 5554 (1973).

${ }^{76} \mathrm{Ch}$. Ackerman, R. Brodman, G. Tolkiehn, G. Zimmerer, R. Haensel, and U. Hahn, J. Luminescence $12 / 13,315$ (1976).

${ }^{77} \mathrm{Ch}$. Ackerman, Thesis, University of Hamburg (1976).

78 R.S. Mulliken, J. Chem. Phys. 52, 5170 (1970); Rad. Res. 59, 357 (1974).

${ }^{79} \mathrm{Y}$. Toyozawa, in Vacuum Ultraviolet Radiation Physics, edited by E.E. Koch, R. Haensel, and C. Kunz, (Pergaman Vieweg, 1974), p. 317.

${ }^{80}$ E. I. Rashba, Izv. USSR Ser. Fiz. 40, 535 (1976). 
${ }^{81}$ K.S. Song, J. Phys. Soc. Japan 26, 1131 (1969).

${ }^{82}$ T. Suemoto, and J. Kanzaki, J. Phys. Soc. Japan 46, 1554 (1979).

${ }^{83}$ U. Hahn, N. Schwentner, and G. Zimmerer, Optics Com. 21, 237 (1977).

${ }^{84}$ Z. Ophir, M.Sc. Thesis, Tel-Aviv University (1970).

${ }^{85}$ R. Englman, and J. Jortner, Mol. Phys. 18, 145 (1970).

${ }^{86}$ N. Schwentner, and E.E. Koch, Phys. Rev. B14, 4687 (1976)

87P.W. Anderson, Phys. Rev. 109, 1492 (1958)

${ }^{88}$ S.K. Lyo, Phys. Rev. B3, 3331 (1973)

89

K. Godzik, and J. Jortner, J. Chem. Fhys. 72, 4471 (1980) 


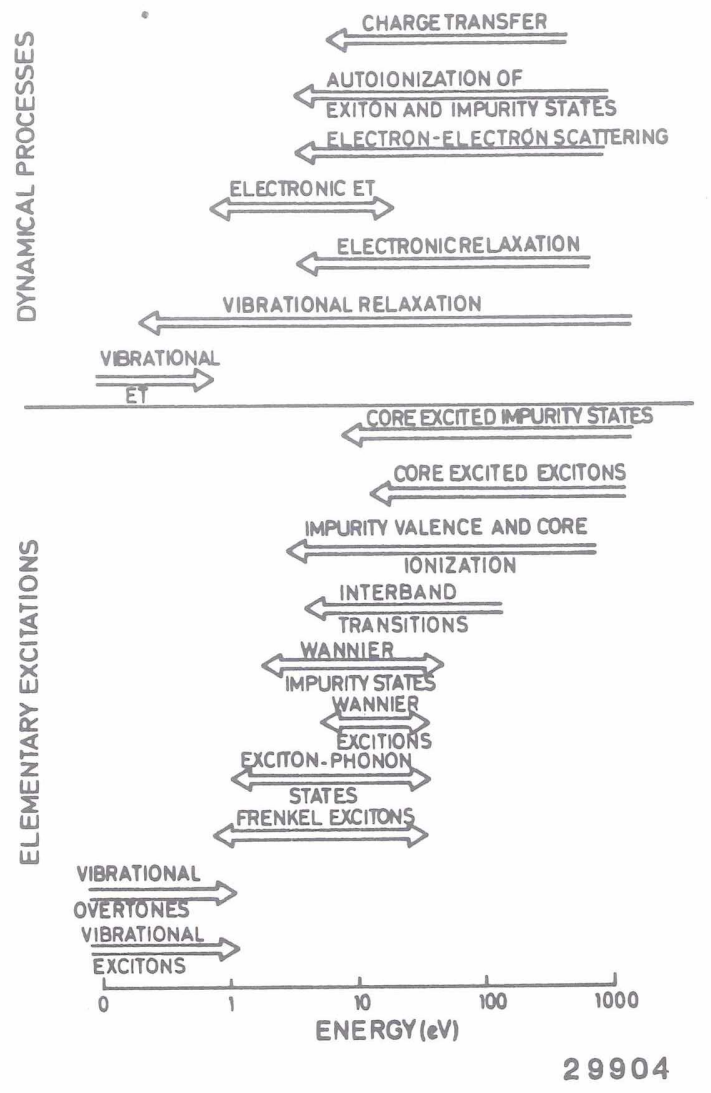

Fig. 1 Energy regions for excited-state photophysical and chemical processes in molecular crystals (after Jortner and Leach, J. Chim. Physique $\mathbf{7 7}$, $7(1980))$.

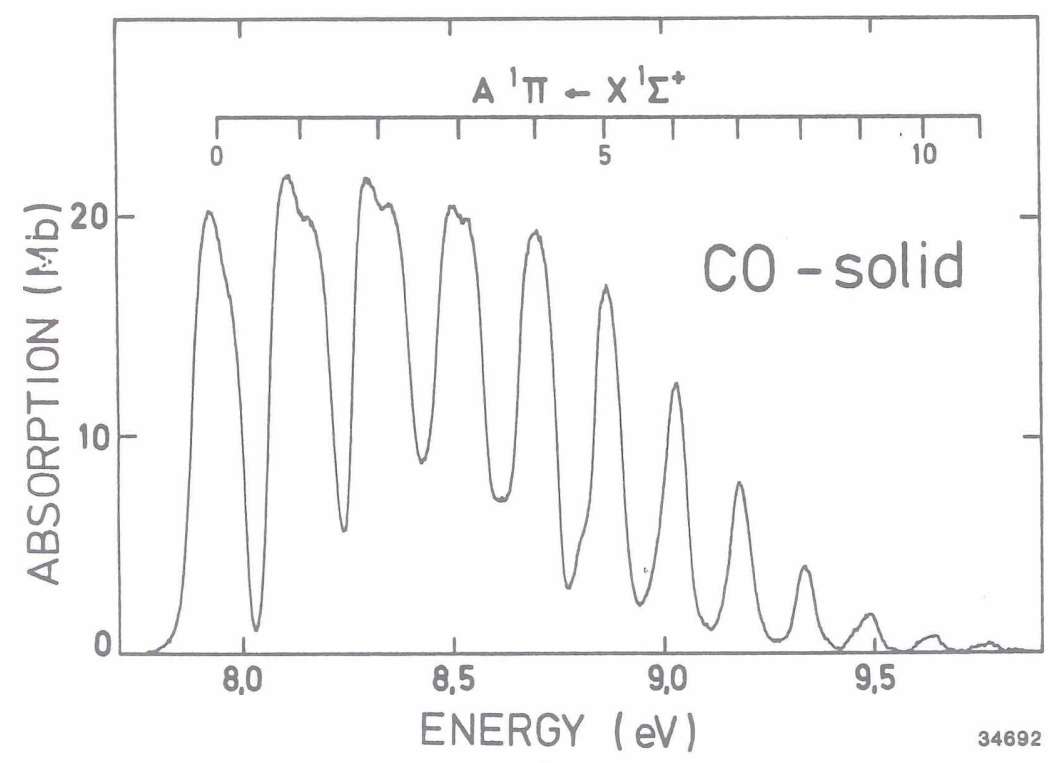

Fig. 2 Absorption spectrum of solid CO in the energy range $7.5-10 \mathrm{eV}$ (from Gürtler, reference 11). 


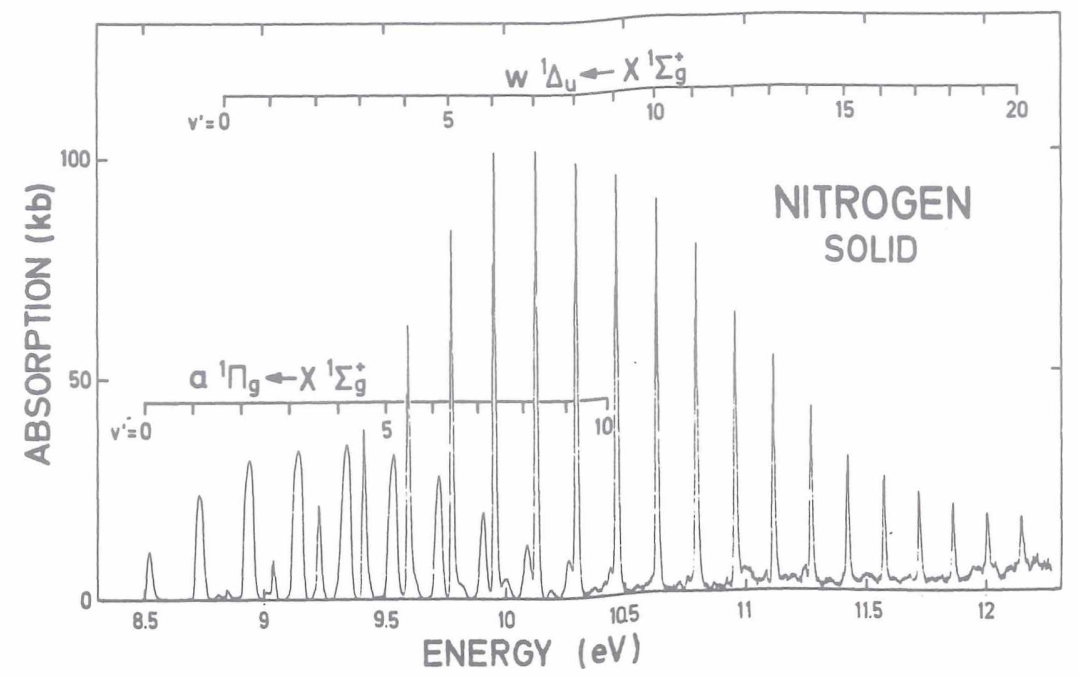

Fig. 3 Absorption spectrum of solid $\mathrm{N}_{2}$ in the energy range $8.0-12.5 \mathrm{eV}$ (from Gürtler, reference 11).

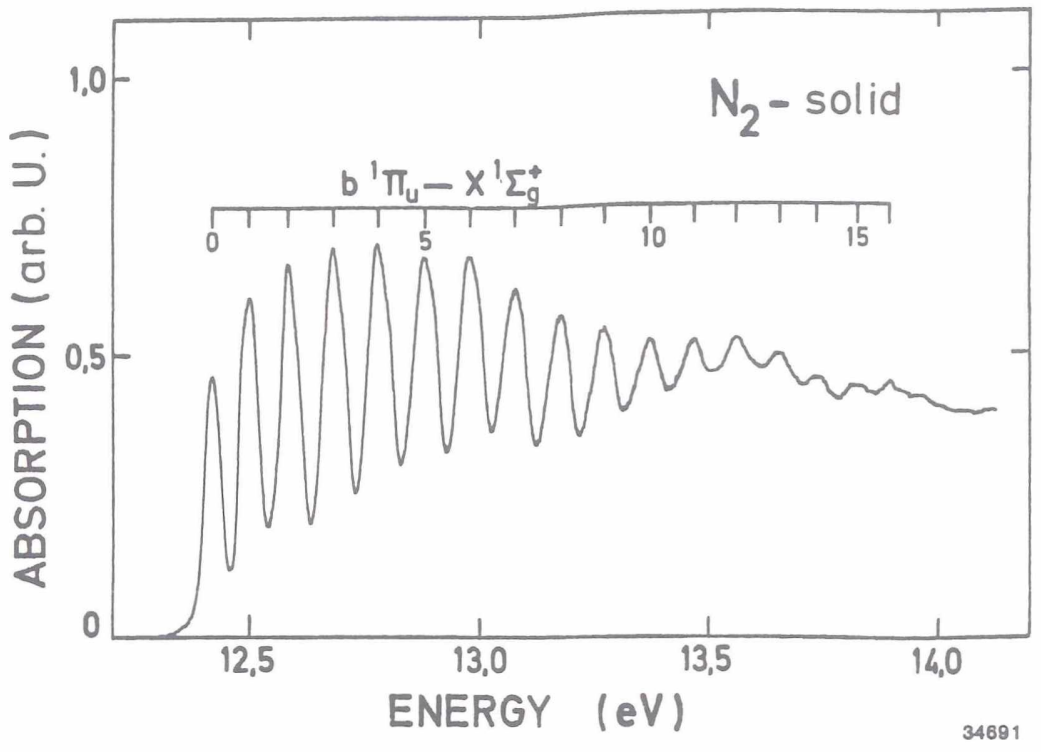

Fig. 4 Absorption spectrum of solid $\mathrm{N}_{2}$ in the energy range $12.0-14.0 \mathrm{eV}$ (from Gürtler, reference 11 and reference 9) 


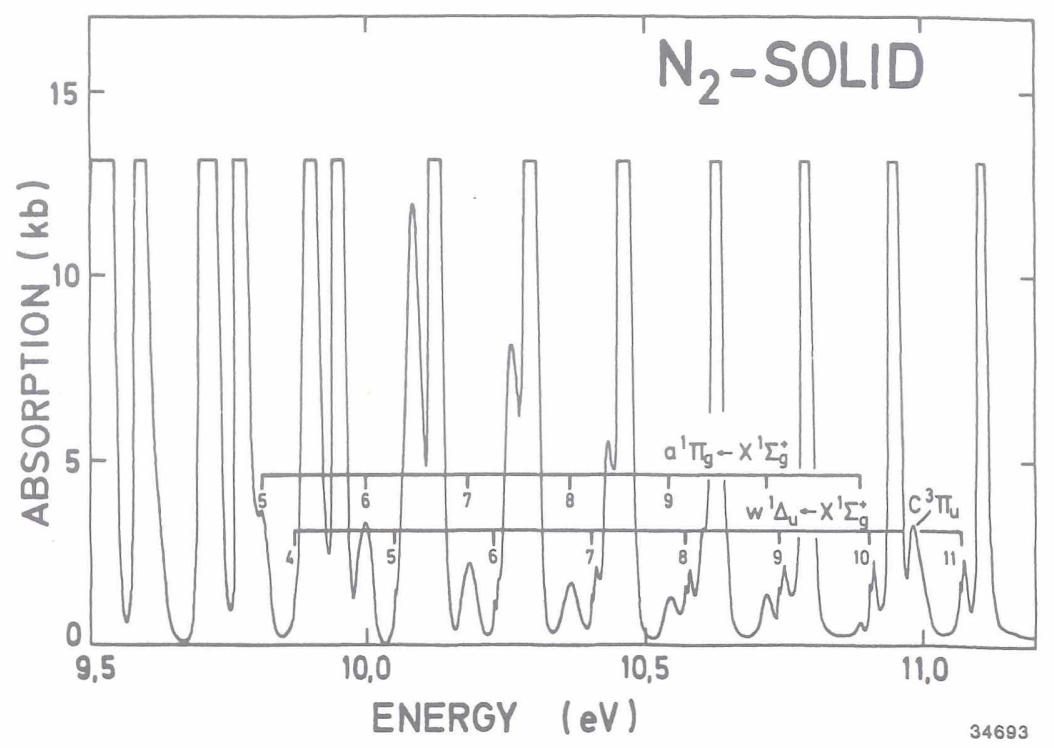

Fig. 5 Cooperative electronic-vibrational excitations in the $x^{\top} \sum_{g}^{+}+w^{1} \Delta_{u}$ transition of solid $\mathrm{N}_{2}$ (after Gürtler, reference 11).

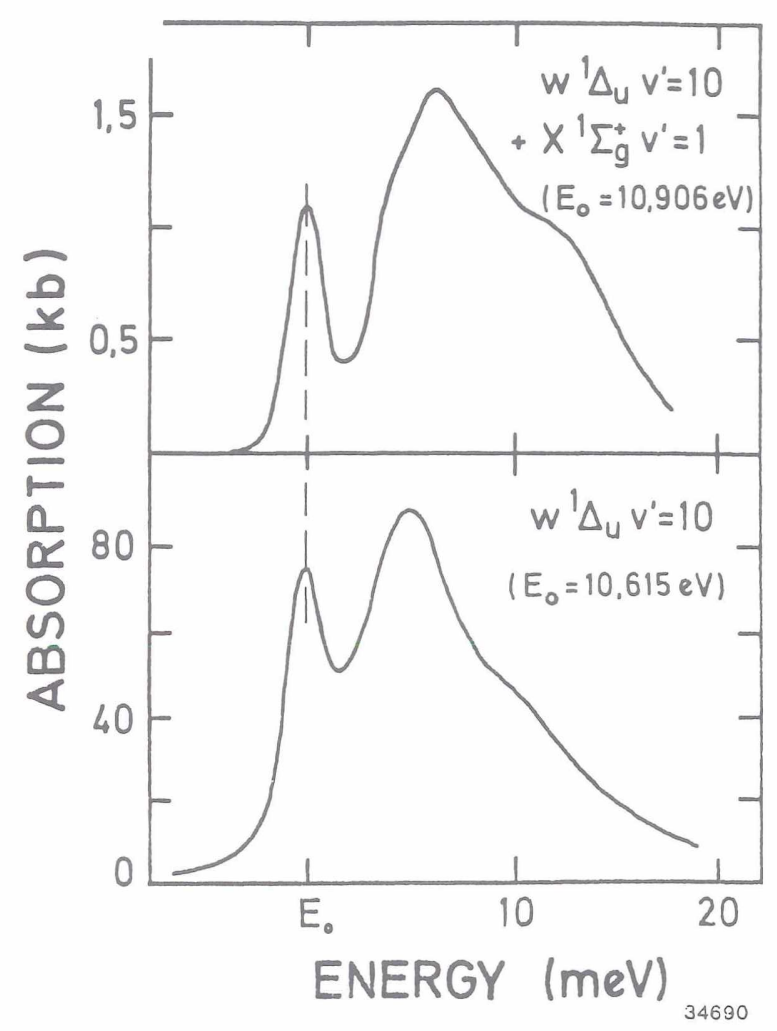

Fig. 6 Lineshapes of one-particle (lower spectrum) and two-particle (upper spectrum) electronic vibrationalexcitationsin solid $\mathrm{N}_{2}$ (after Gürtler, reference 11). 


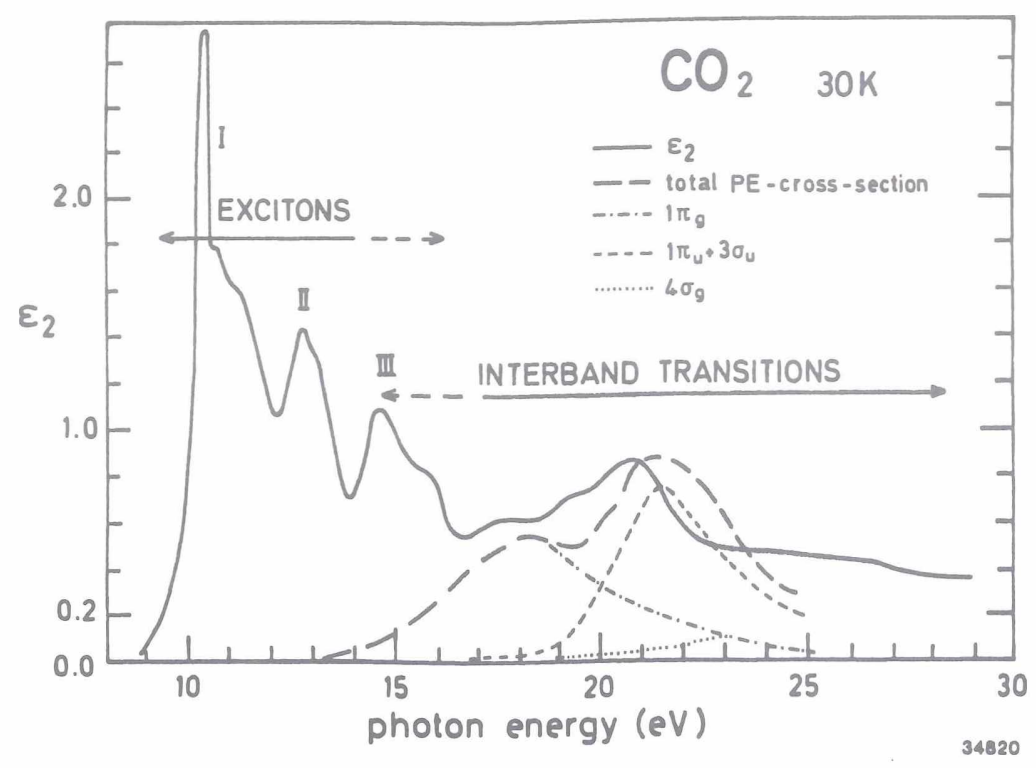

Fig. 7 Optical absorption spectrum (solid curve) and photoelectron cross sections of solid $\mathrm{CO}_{2}$ in the energy ranges 9-30 eV (from Fock, Lau, and Koch, references 18 and 19).

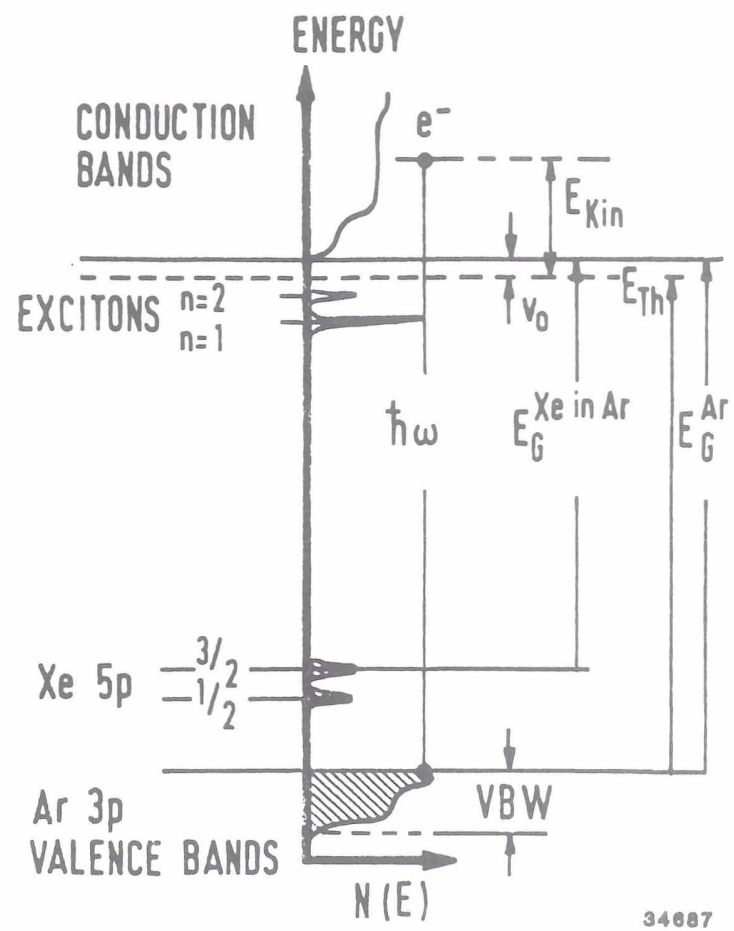

Fig. 8 Schematic scheme of the energy levels of excitons and atomic impurity states in a solid rare gas matrix depicted for the case of $\mathrm{Xe}$ in $\mathrm{Ar}$. 


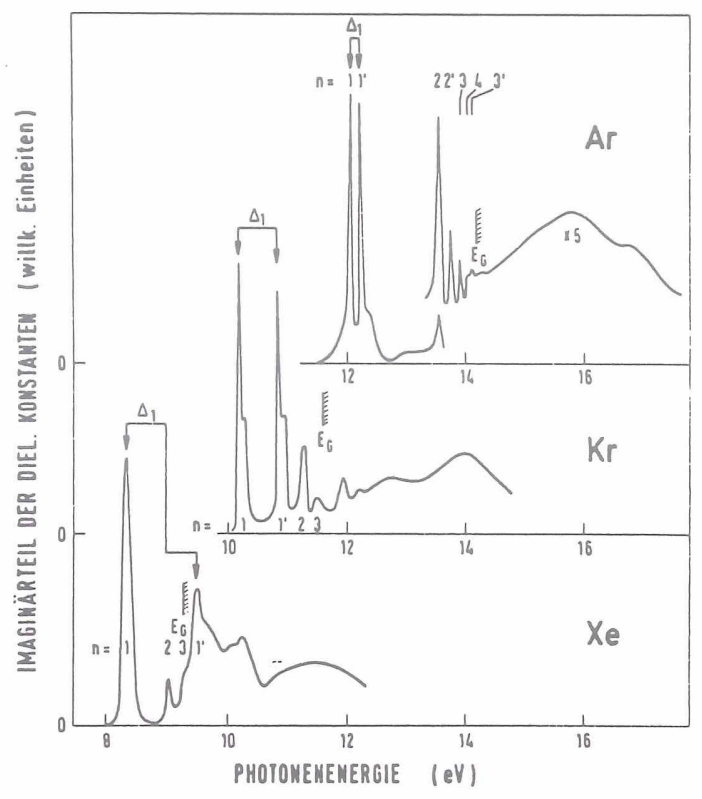

Fig. 9 The spectra of the imaginary part of the dielectric constant $\varepsilon_{2}$ for solid $\mathrm{Ar}, \mathrm{Kr}$, and $\mathrm{Xe}$ in the region of the valence excitons. The spin orbit splitting in the $n=1$ excitons $\Delta y$, the assignments of the higher members of the exciton series and the band gap $E_{G}$ are indicated. 


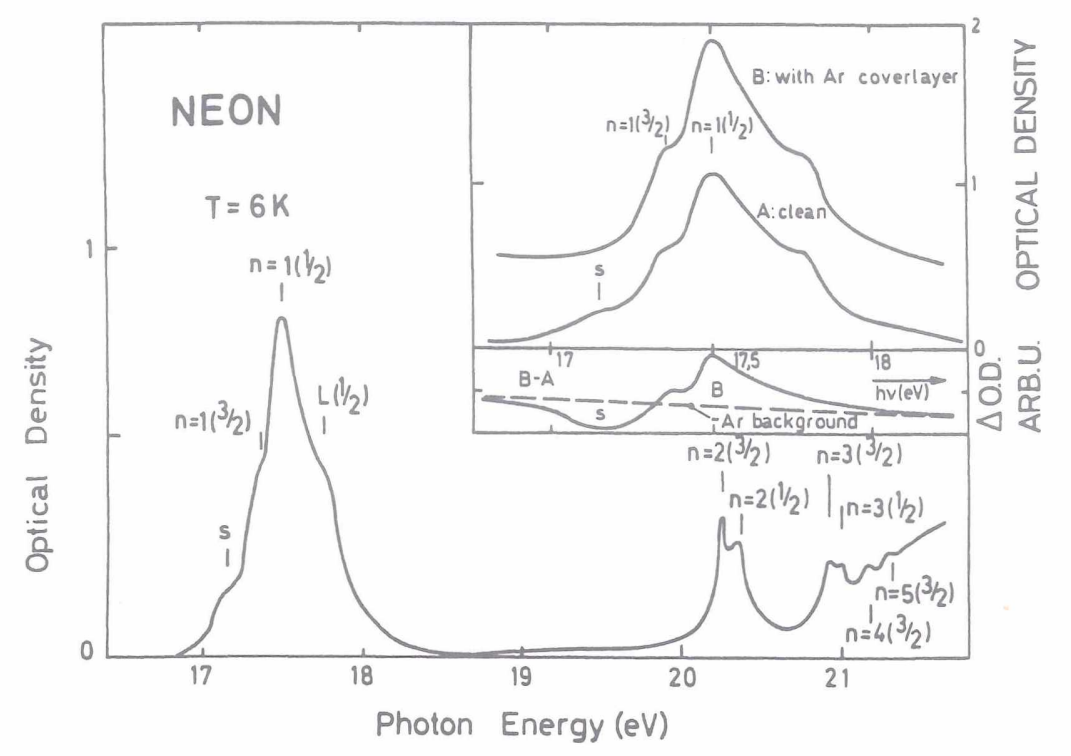

Fig. 10 Optical density of solid neon at $6 \mathrm{k}$ in the excitonic range of the spectrum. The main features can be grouped into two series split by spin orbit interaction which converge to the bandgap. In the insert results of a surface coverage experiment for solid neon in the range of the $n=1$ exciton are shown. Curve $A$ is the optical density of a clean neon sample. Upon evaporation of a thin Ar coverlayer the structure denoted by S disappears, whereas the band shape of the remaining peak remains unchanged. In the lower part of the insert, the difference spectrum B-A is shown and the smooth and structureless Ar background is indicated by the broken line (from Saile and Koch, ref. 27). 


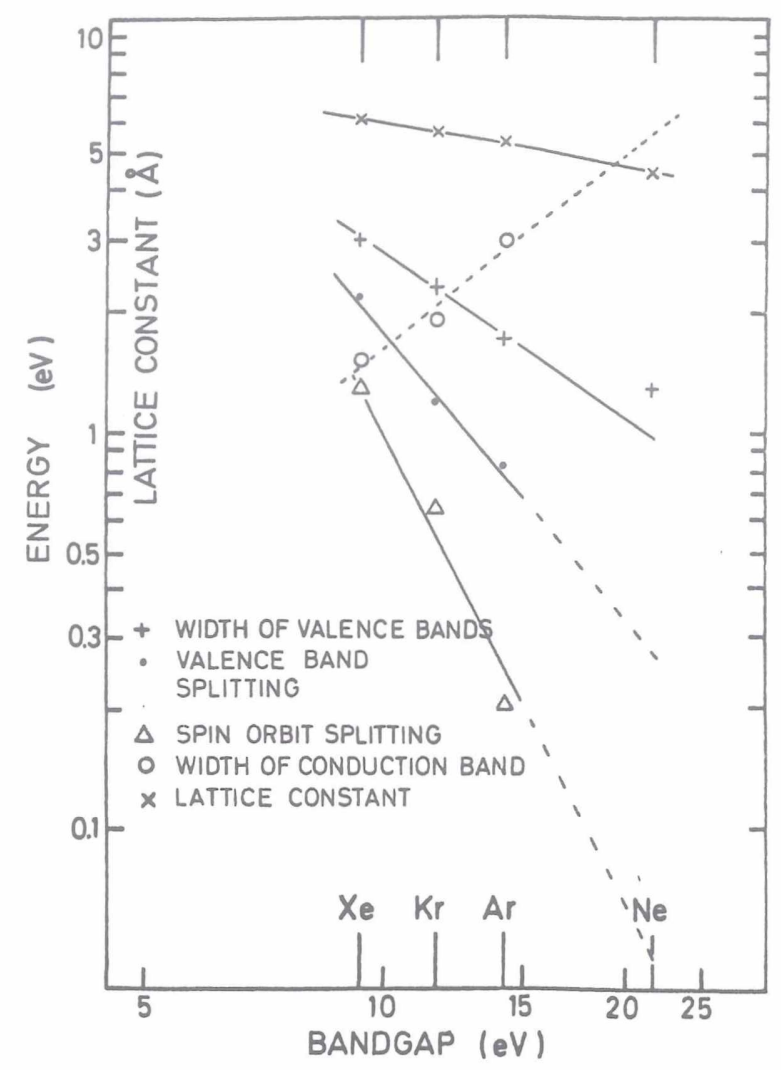

Fig. 11 The plot of characteristic parameters of the valence and conduction bands $(+$, width of valence bands; $\bullet$, valence band splitting; $\Delta$, spin orbit splitting; 0 , width of conduction band; $x$, lattice constant) on a linear band gap scale shows a continuous change of these parameters from Xe to $\mathrm{Ne}$ (after Schwentner, thesis; University of München, 1974). 


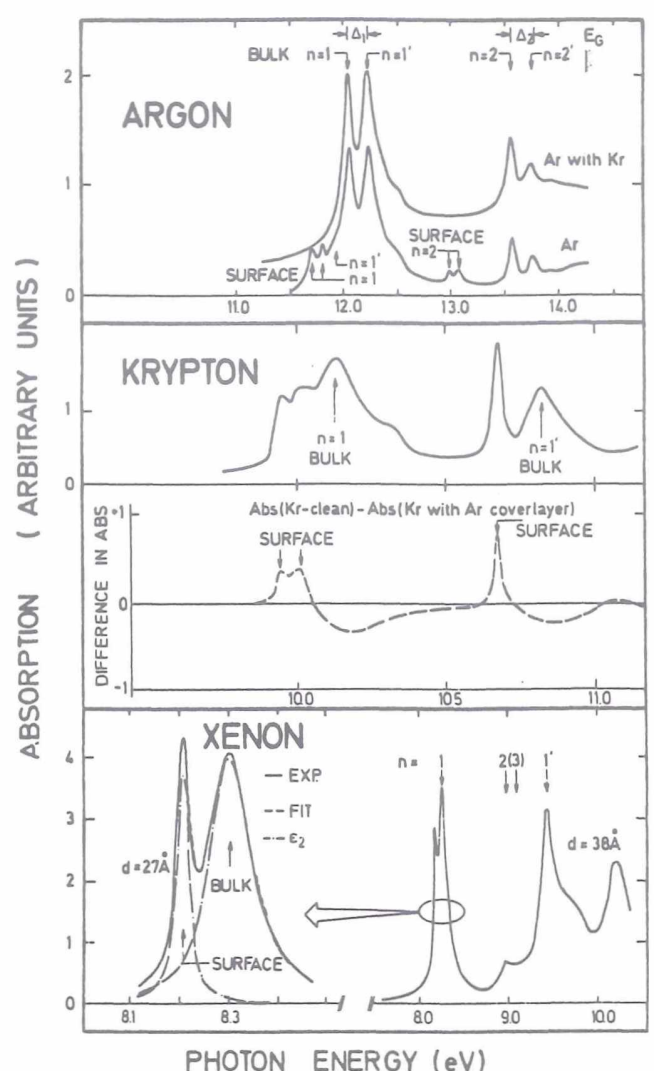

Fig. 12 High resolution absorption spectra for solid $\mathrm{Ar}, \mathrm{Kr}$ and $\mathrm{Xe}$ in the range of the valence excitons. Volume and surface excitons are observed for all three samples. For $\mathrm{Ar}$ and $\mathrm{Kr}$ the results of surface coverage experiments are also shown. For Xe the experimentally determined spectrum in the range of the $n=1$ surface and volume exciton is displayed on an expanded scale together with a lineshape analysis using two Lorentzians (after Saile, thesis, University of München, 1976). 


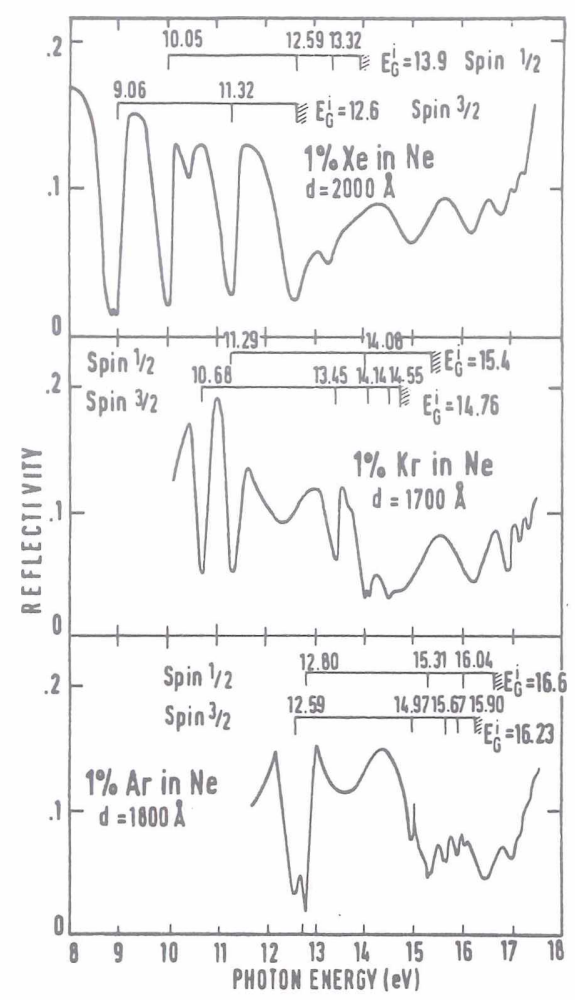

Fig. 13 Reflectance spectra of Ne films doped with $1 \% \mathrm{Xe}, d=2000 \AA$ (upper panel); $1 \% \mathrm{Kr}, \mathrm{d}=1700 \AA$ (middle panel); and $1 \% \mathrm{Ar}, \mathrm{d}=1800 \AA$ (lower panel) (d: film thickness) evaporated onto a gold substrate. The spin-orbit-split impurity exciton series are indicated on top of each spectrum. (From Pudewill et al., reference 40 ). 


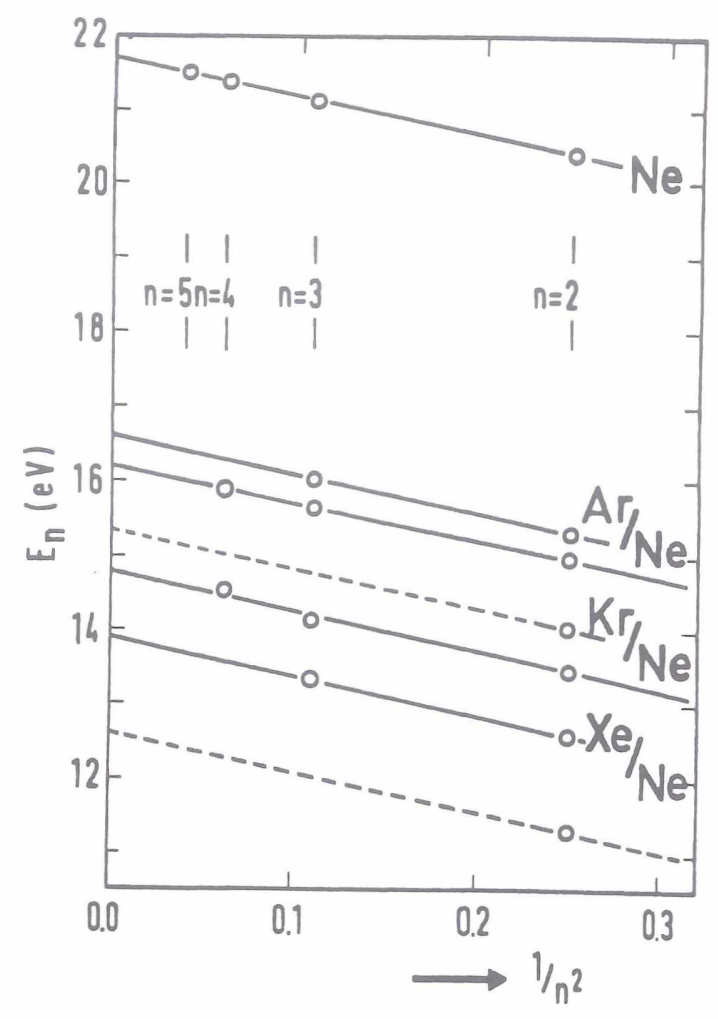

Fig. 14 Excitation energies of excitons in pure $\mathrm{Ne}\left(E_{n}\right)$ and of the impurity states of $\mathrm{Ar}, \mathrm{Kr}$ and $\mathrm{Xe}$ in a Ne matrix $\left(E_{n}{ }^{i}\right)$ plotted versus $1 / n^{2}$ according to the Wannier model. (From Pudewill et al., reference 40.)

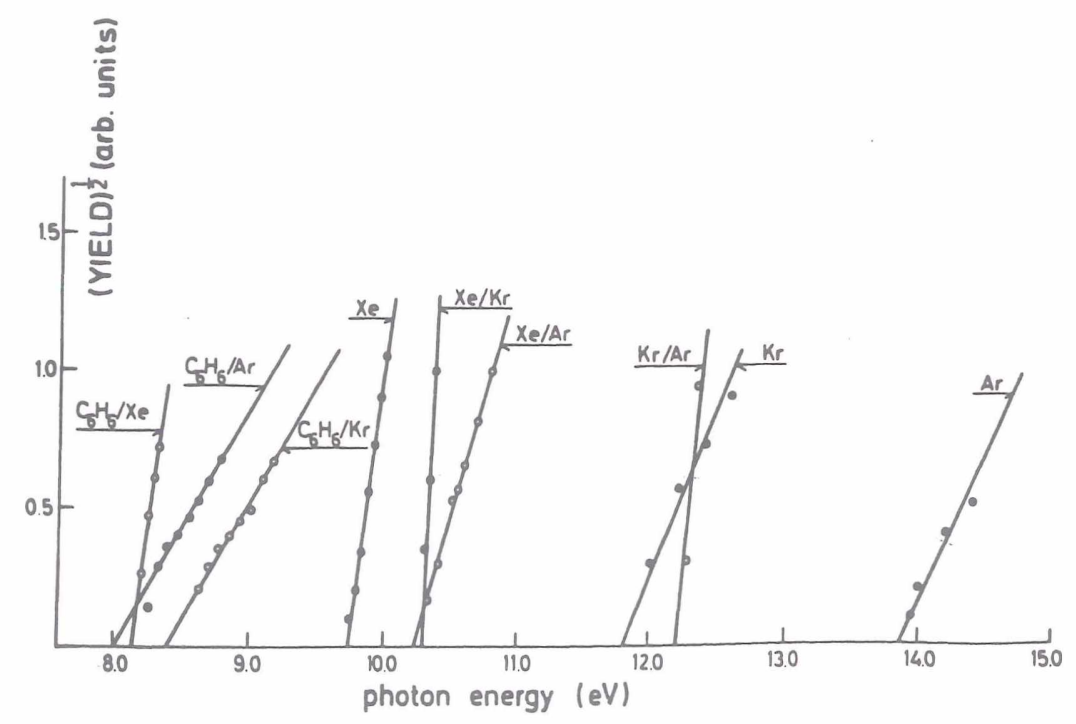

Fig. 15 Photoemission onsets from pure rare gases and from impurity states in RGS (reference 57). 


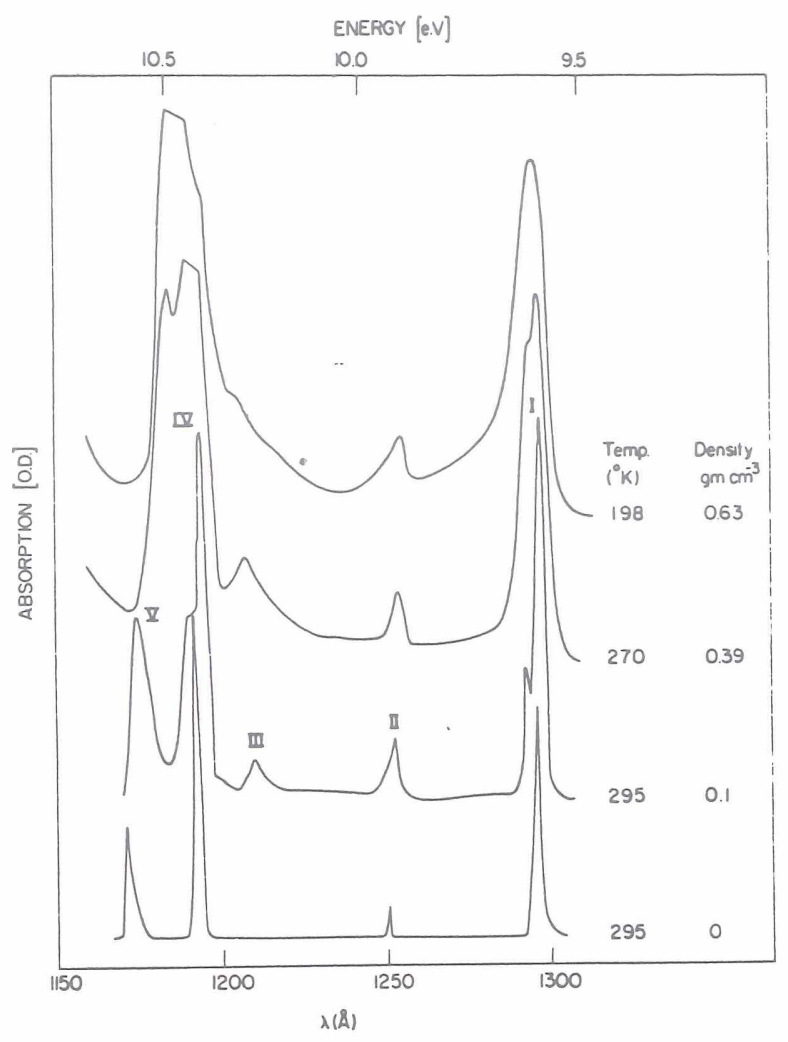

Fig. 16 The absorption spectra of Xe in fluid Ar in the low and intermediate Ar density range. The absorption curves are horizontally shifted, all being displayed on the same optical density (OD) scale. (From Messing et al., ref. 50.) 


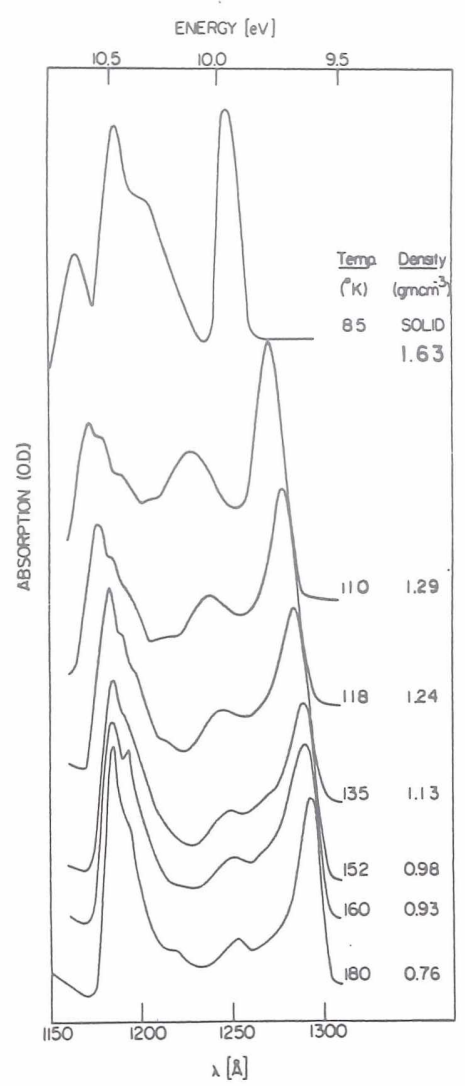

Fig. 17 The absorption spectra of Xe in fluid Ar at high Ar density and in solid Ar. The Xe concentration is roughly $1 \mathrm{ppm}$. The absorption curves are horizontally shifted, all being displayed on the same optical density (OD) scale. (From Messing et al., ref. 50.) 


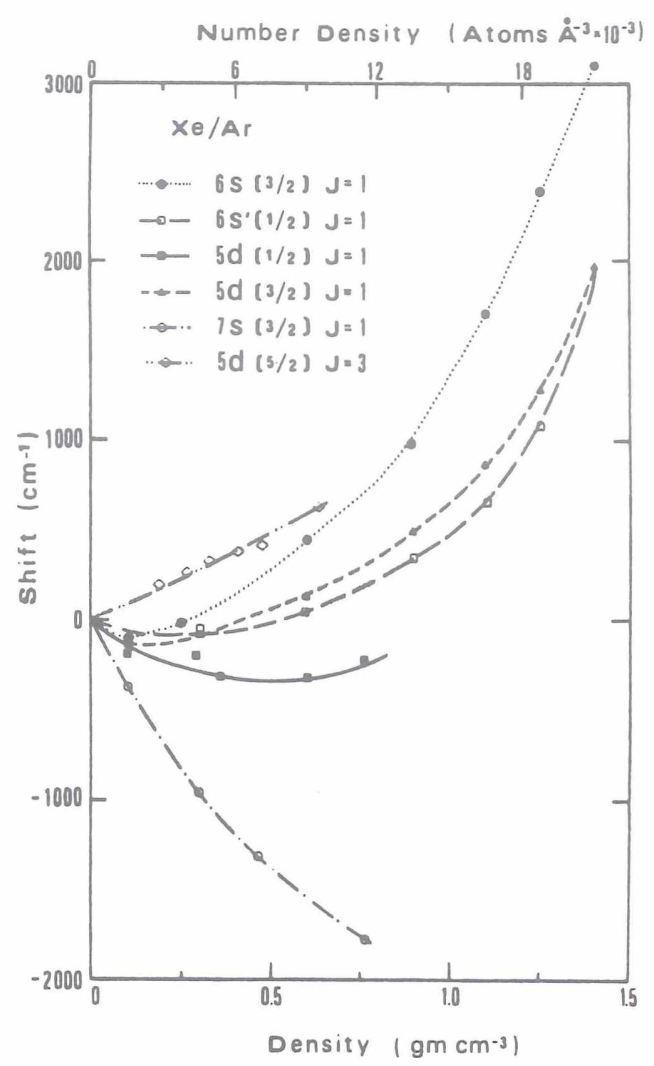

Fig. 18 The density dependence of the energies of the maxima of the absorption bands relative to the unperturbed atomic transition energy for the absorption lines of $\mathrm{Xe}$ in fluid Ar observed in the spectral range $8.5 \mathrm{eV}(\simeq 1460 \AA)$ to $10.8 \mathrm{eV}(1150 \AA)$; see Figs. 16 and 17 . The spectral lines are designated by the conventional atomic notation. (From Messing et al., ref. 50. ) 


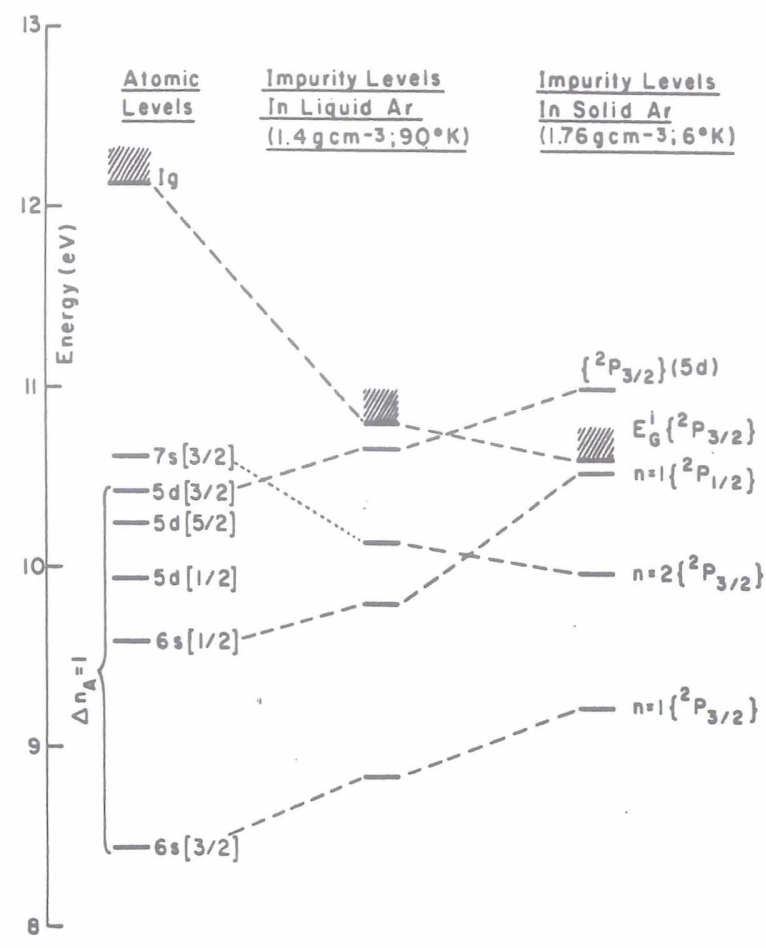

Fig. 19 Energy correlation diagram of the unperturbed atomic states of $\mathrm{Xe}$, Xe impurity states in liquid Ar and of Xe impurity states in solid Ar. The correlation of the $7 \mathrm{~s}$ [3/2] atomic state is rather tentative as denoted by the dashed line. (From Messing et al., ref. 50.) 


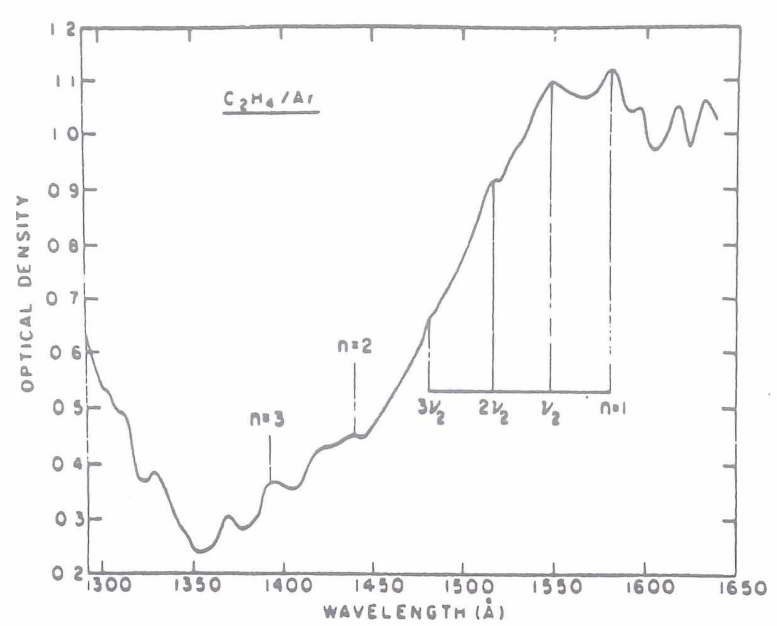

a)

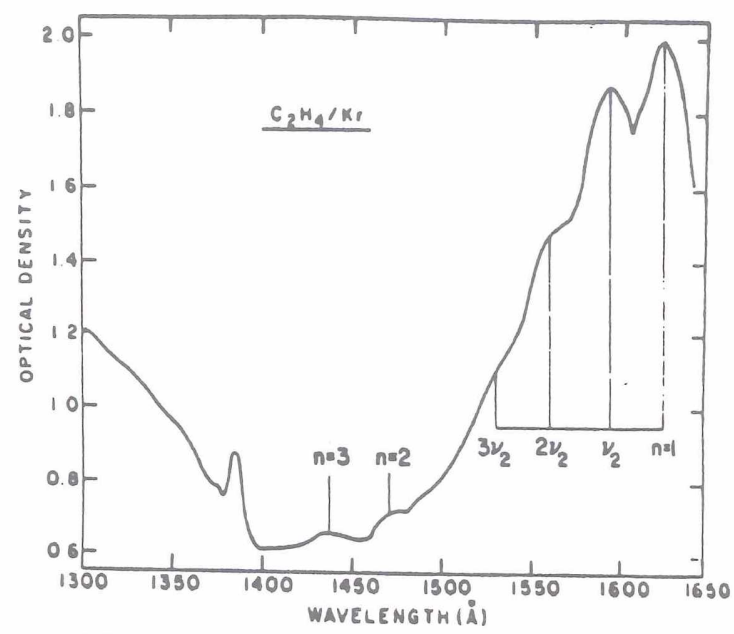

b)

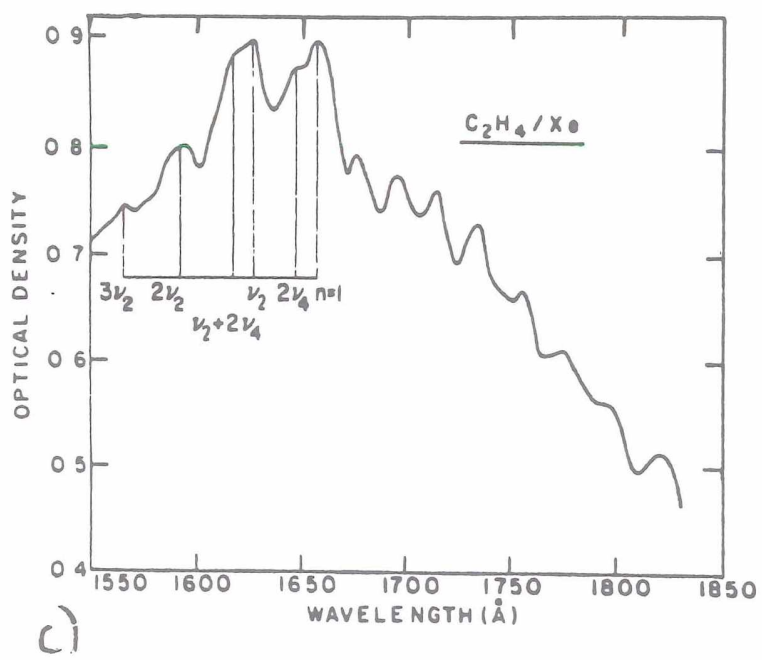

Fig. 20 Extravalence electronic excitations of ethylene in RGS (from Gedanken et al., ref. 39). 


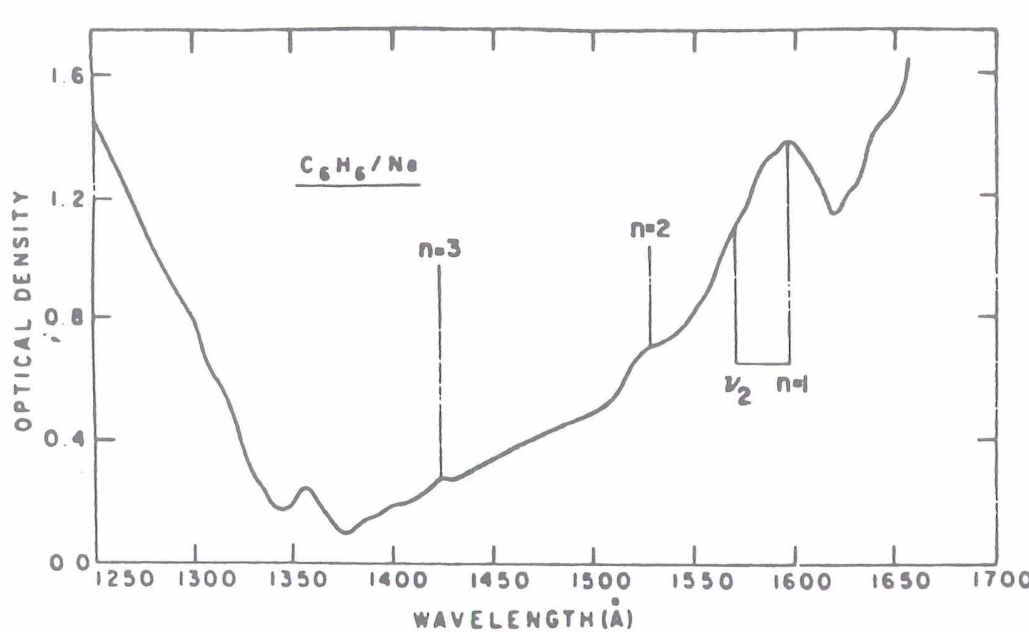

a)

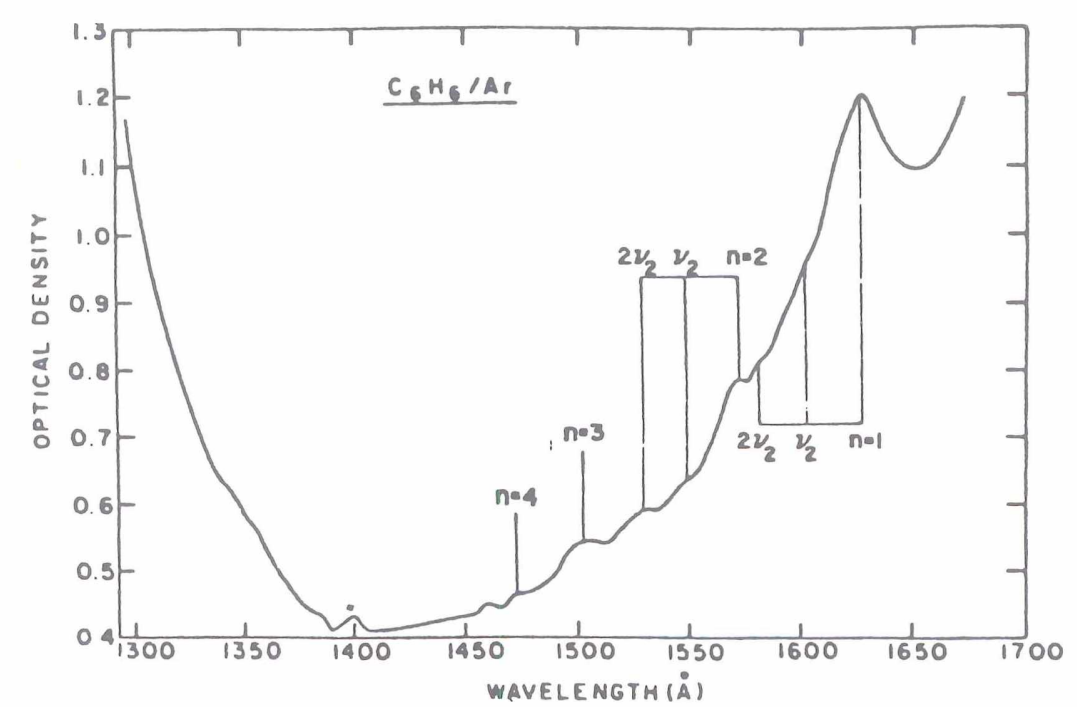

b)

d)

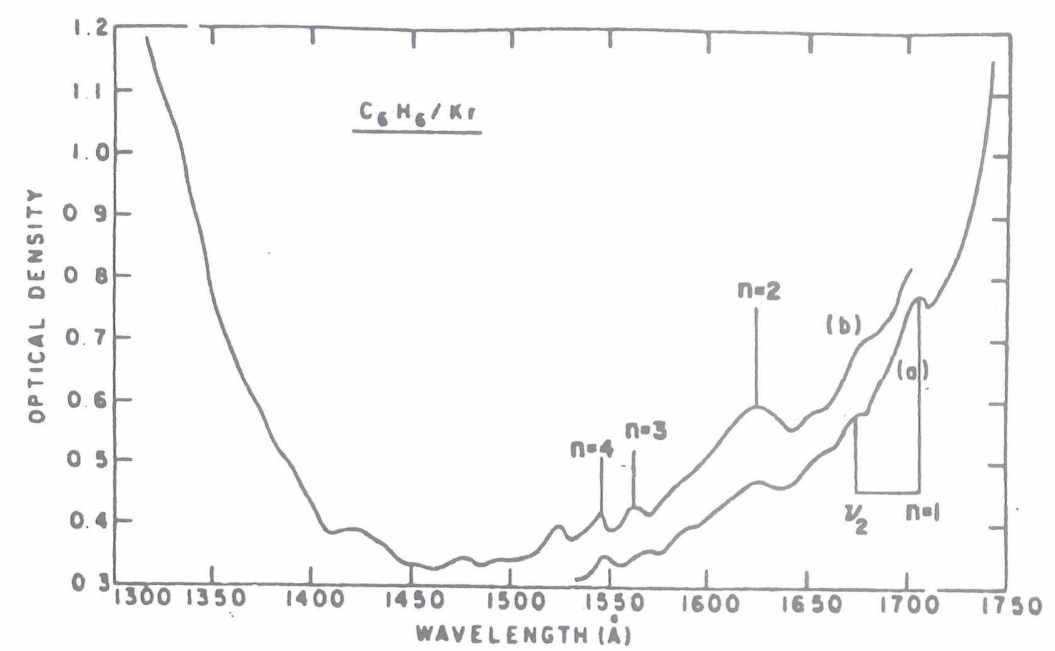

c)

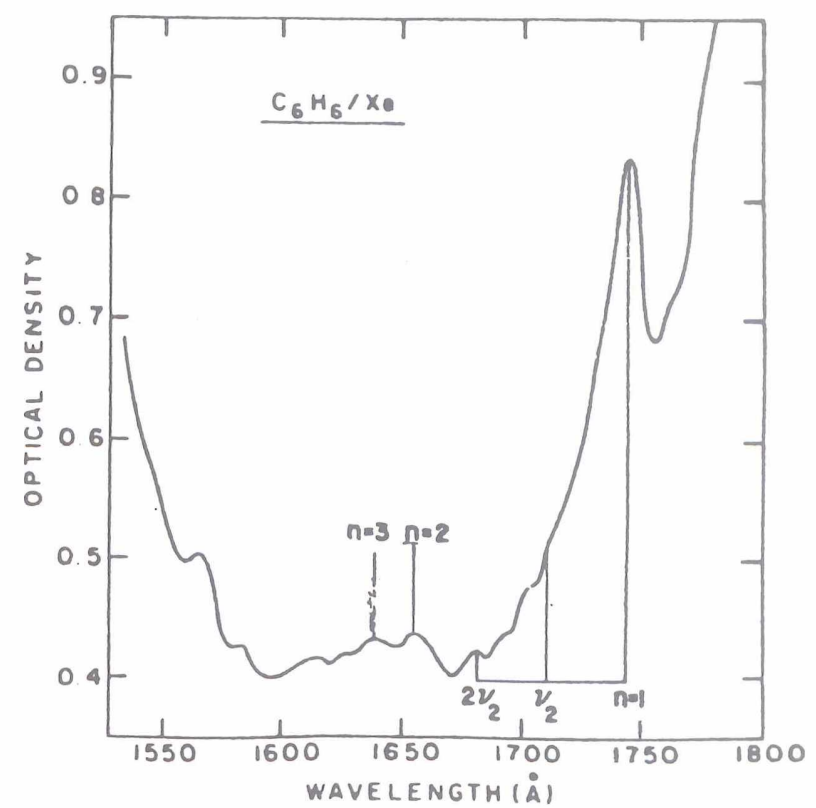

Fig. 21 Extravalence electronic excitations of benzene in insulators (from Gedanken et a1., ref. 39). 


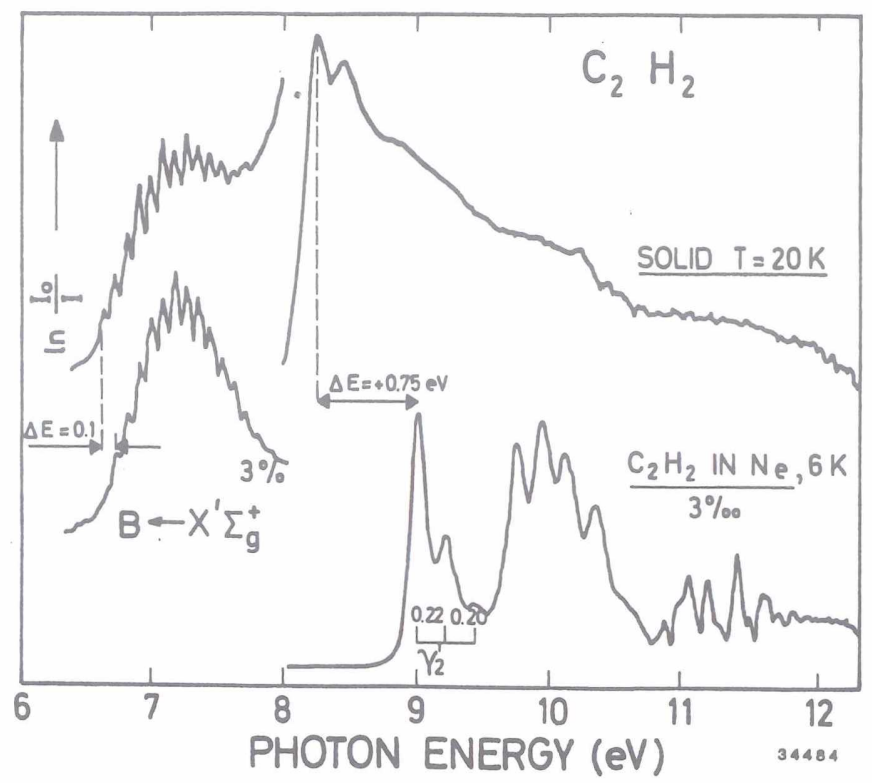

Fig. 22 The absorption spectra of solid acetylene and of acetylene in solid $\mathrm{Ne}$ (from E.E. Koch, and J.H. Fock, ref. 59).

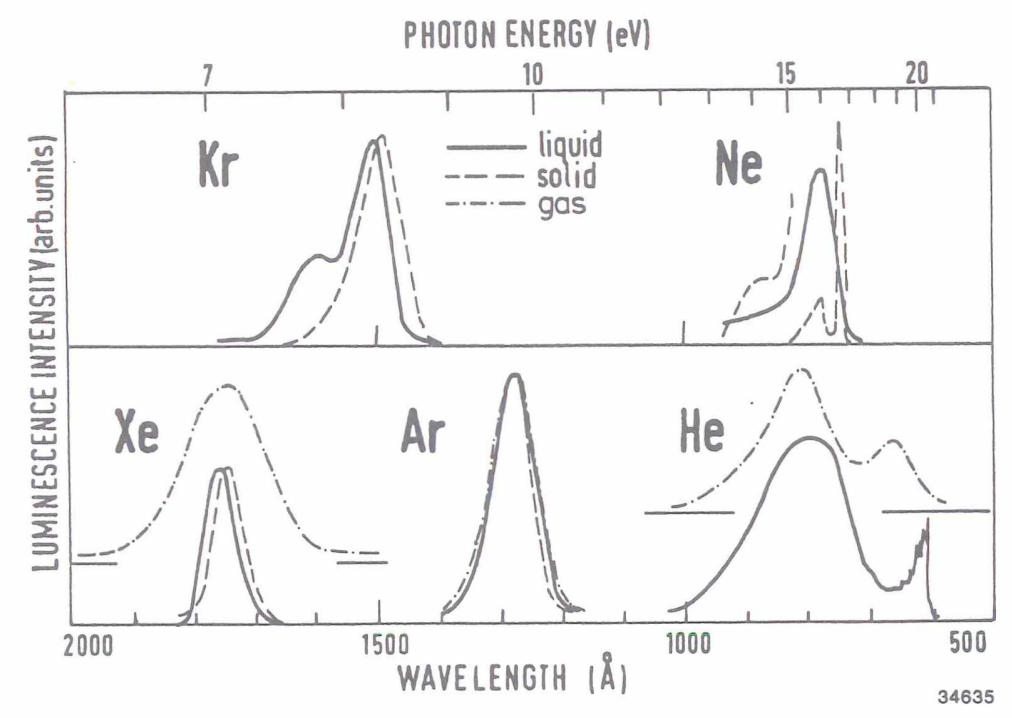

Fig. 23 Emission spectra of solid, liquid and gas-phase rare gases. 


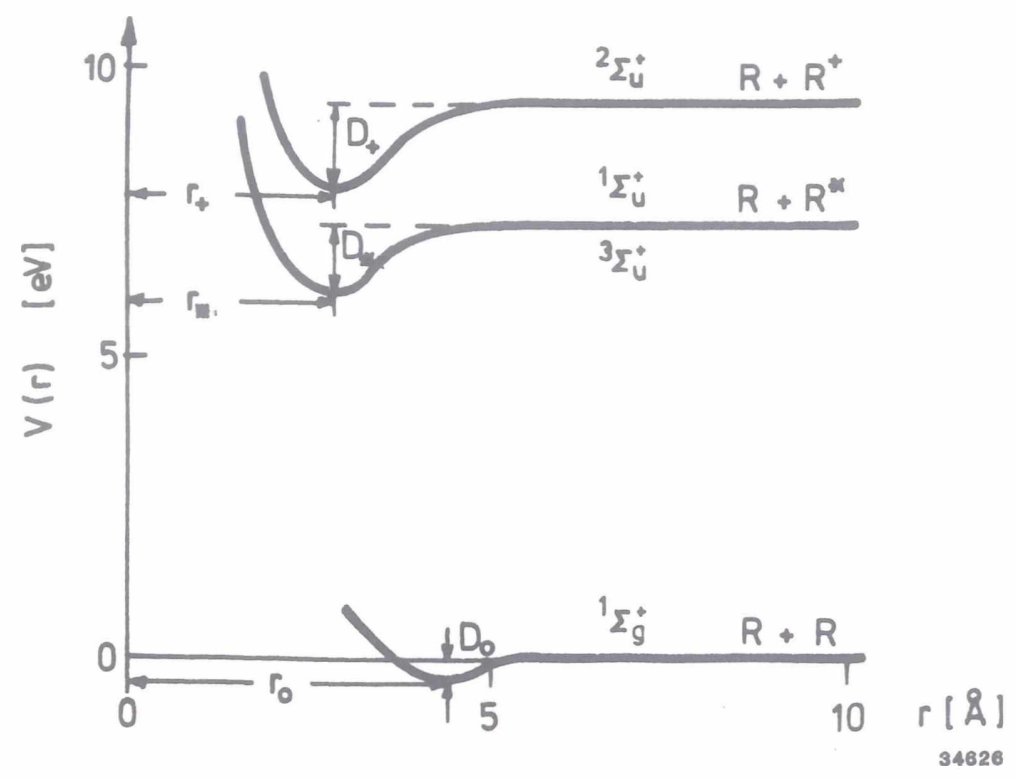

Fig. 24 Potential curves for a diatomic rare gas molecule.

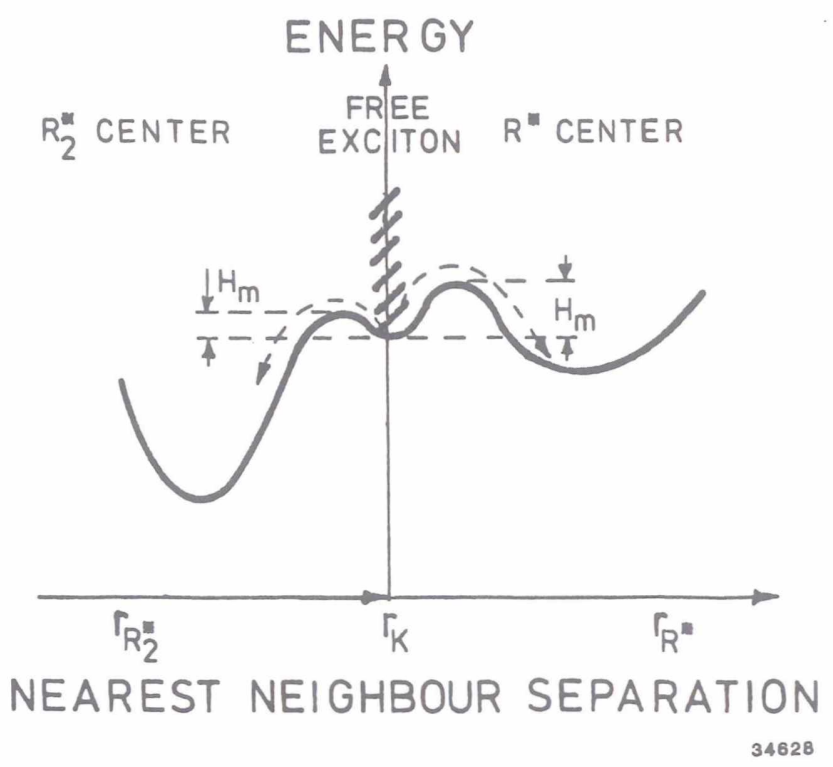

Fig. 25 Schematic potential curves and lattice distortions describing the localization of free excitons in atomic and excimer centers (after Fugol, Advances in Phys. 27, 1 (1978)). 

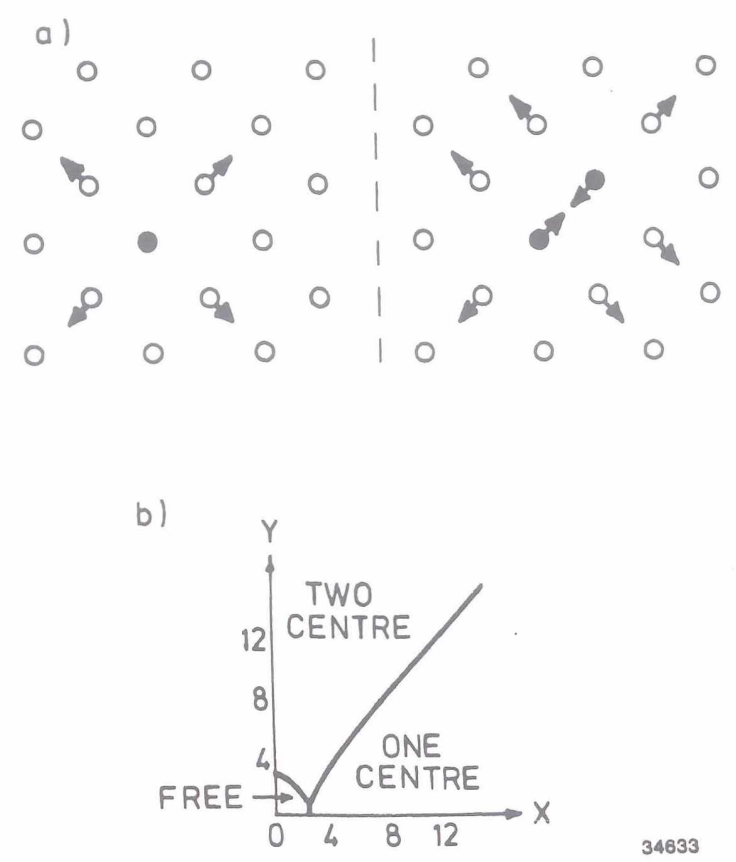

Fig. 26 a) Schematic lattice rearrangement for one center selftrapping (1eft) and two center selftrapping (right) of excitons in RGS after Song, (ref. 81) and Jortner (ref. 22).

b) Map of zones of stable states of selftrapper excitons in the plane $X=U^{\prime} / \sqrt{\alpha|V|}, Y=V^{\prime} / \sqrt{\alpha \mid V T}$ (from Song, ref. 81).

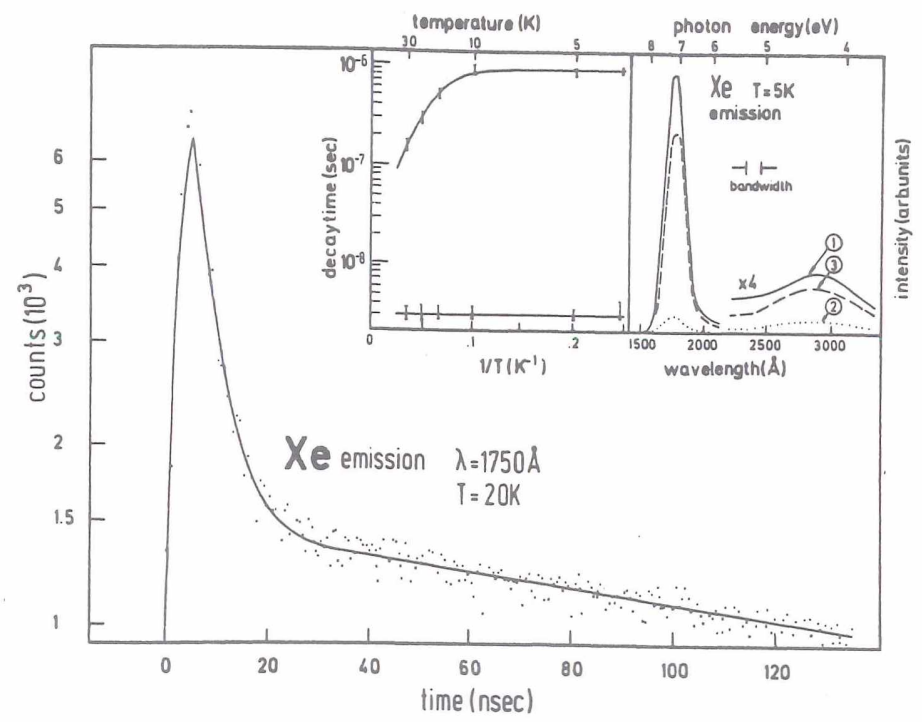

Fig. 27 Decay curves for solid Xe. Left insert, temperature dependence of the long and short components; right insert, emission bands for different times after deposition of the sample (Hahn et al., ref.83). 


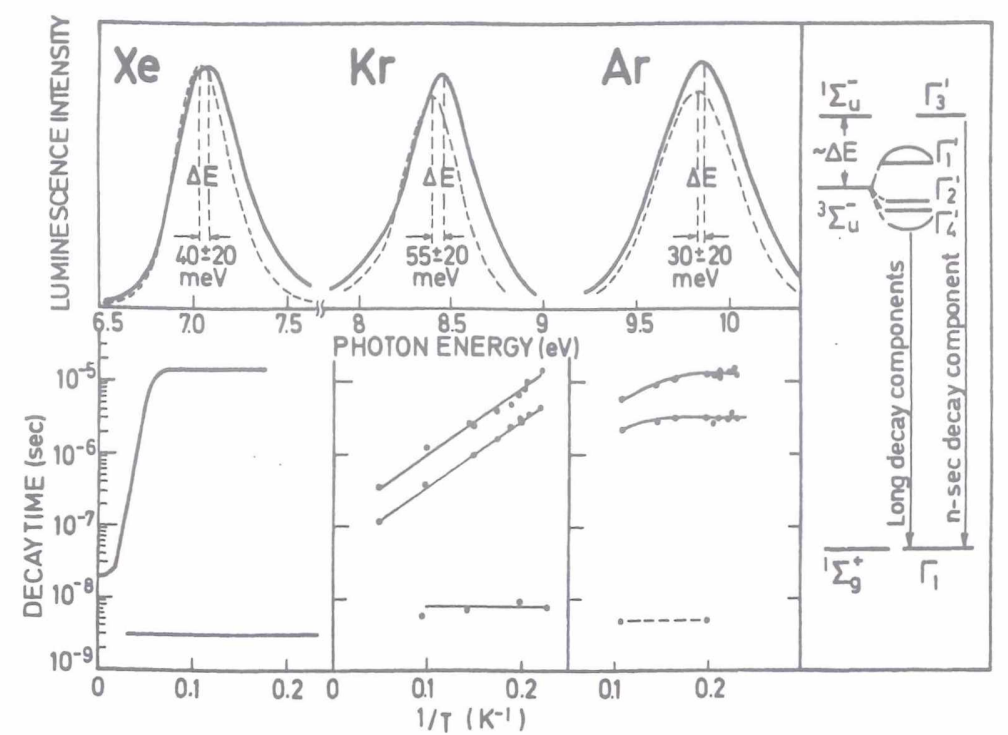

Fig. 28 Results of time-resolved luminescence experiments for the main luminescence bands of solid $\mathrm{Xe}, \mathrm{Kr}$ and Ar. Full lines in the intensity curves (upper panel) correspond to the component with short decay times $\left(10^{-9} \mathrm{~s}\right.$ region), dashed lines to long decay times. Emission bands and decay curves for $\mathrm{Kr}$ and $\mathrm{Ar}$ are obtained by Hahn et a1. (from G. Zimmerer, J. Luminescence 18/19,875(1979)

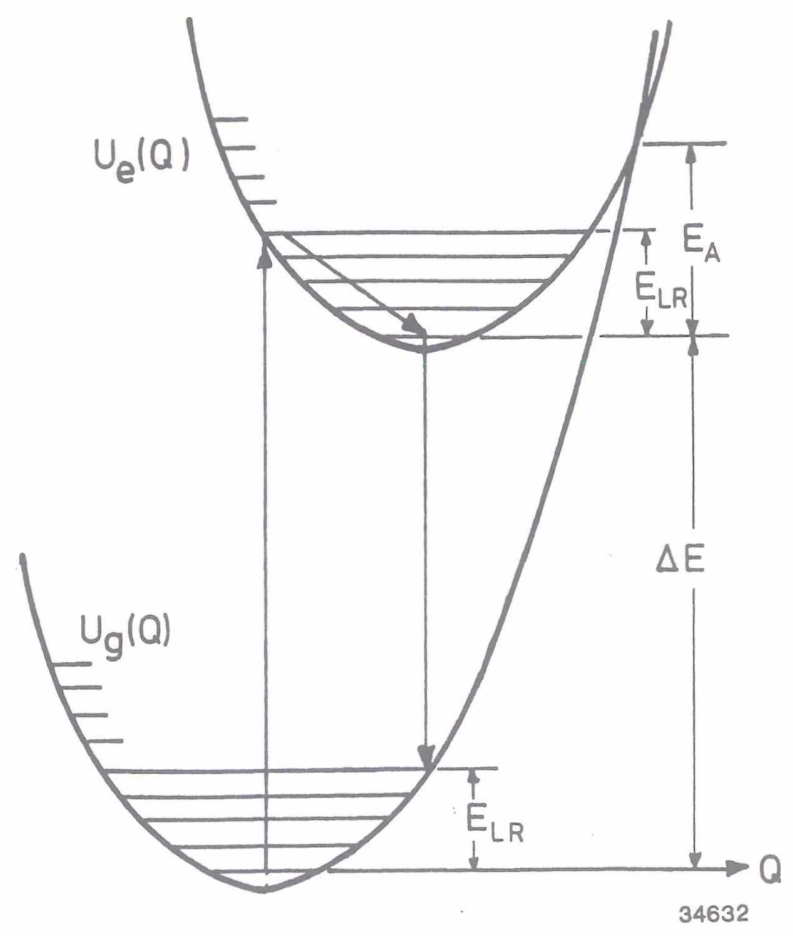

Fig. 29 A configurational diagram for an impurity center. 


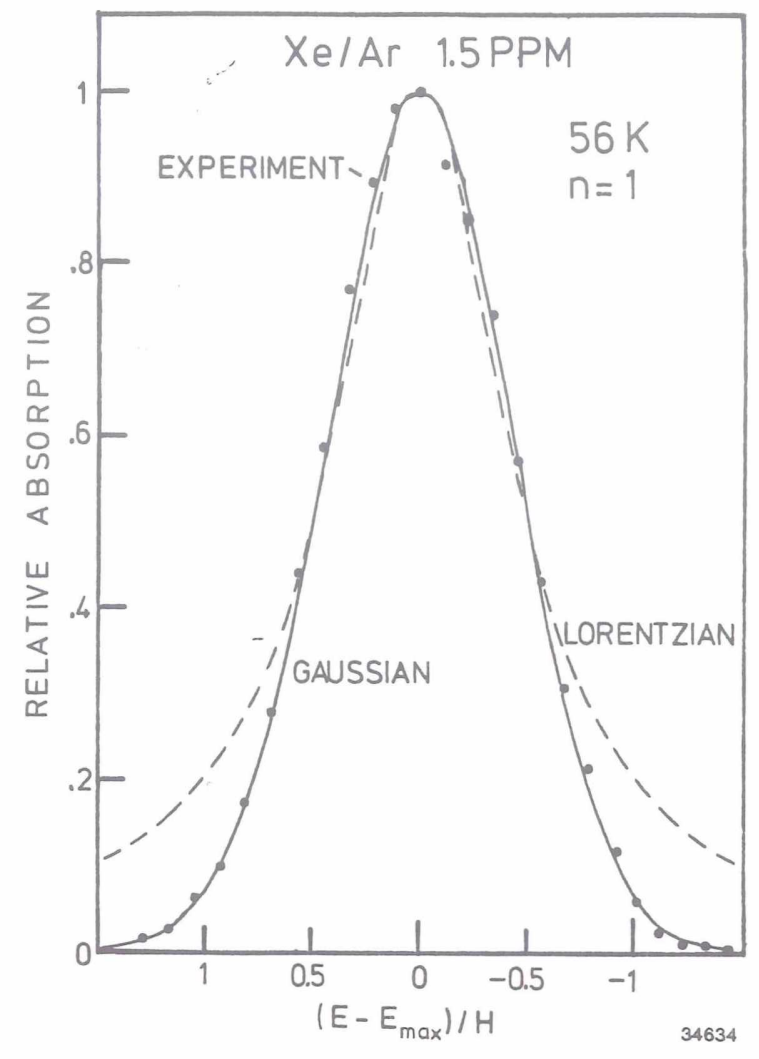

Fig. 30 Experimental absorption lineshape (points) of the $n=1$ exciton of Xe guest atoms in an Ar crystal at $56 \mathrm{~K}$ and fits with a Gaussian (solid line) and Lorentzian (dashed line) lineshape. The center of the line $E_{\max }$ corresponds to $9.156 \mathrm{eV}$ and the halfwidth (fwhm) $H$ is $119 \mathrm{meV}$. (After Ophir, ref. 84.) 

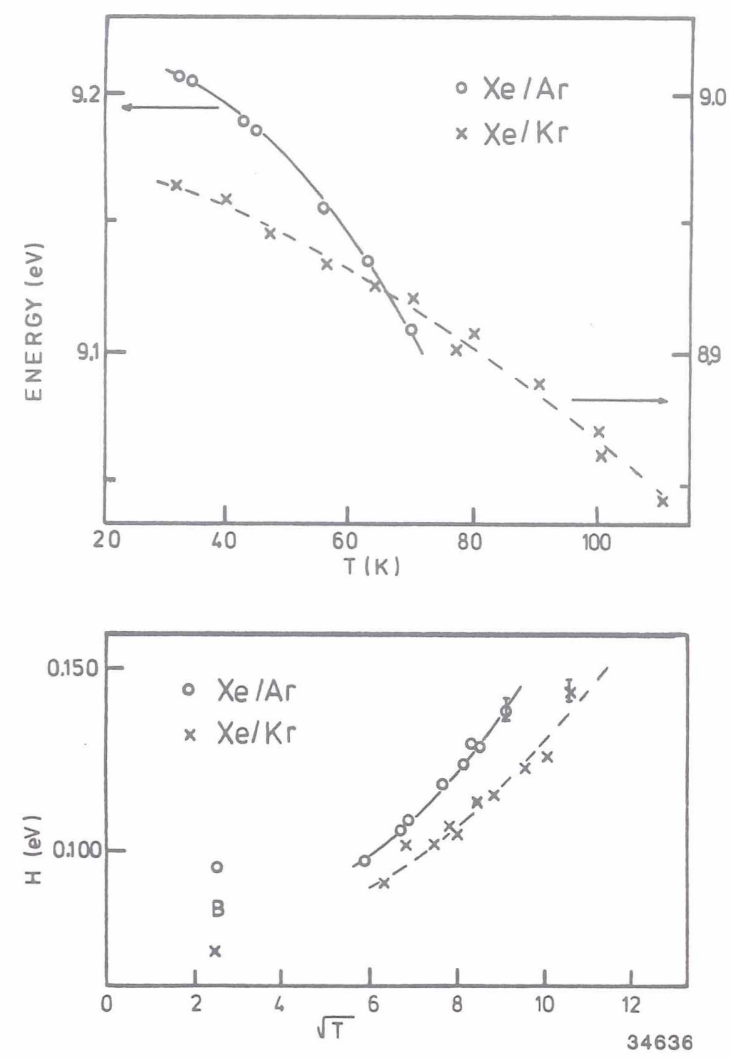

Fig. 31 Temperature dependence of the position of the $n=1$ excitons of $\mathrm{Xe}$ guest atoms in $\mathrm{Ar}$ (circles) and $\mathrm{Xe}$ guest atoms in $\mathrm{Kr}$ crystals (crosses) (upper part) and of the corresponding halfwidth $\mathrm{H}$ (lower part). The result of Baldini (1965) is shown by $B$. The solid (Ar matrix) and dashed ( $K r$ matrix) lines correspond to fits with equ. 9.1 (1ower part) and $E_{\max }=E_{\max }(0)+\hbar \Delta \omega \operatorname{coth}\left(\frac{\hbar<\omega>}{2 k T}\right)$ (upper part). (After Ophir, ref. 84). 


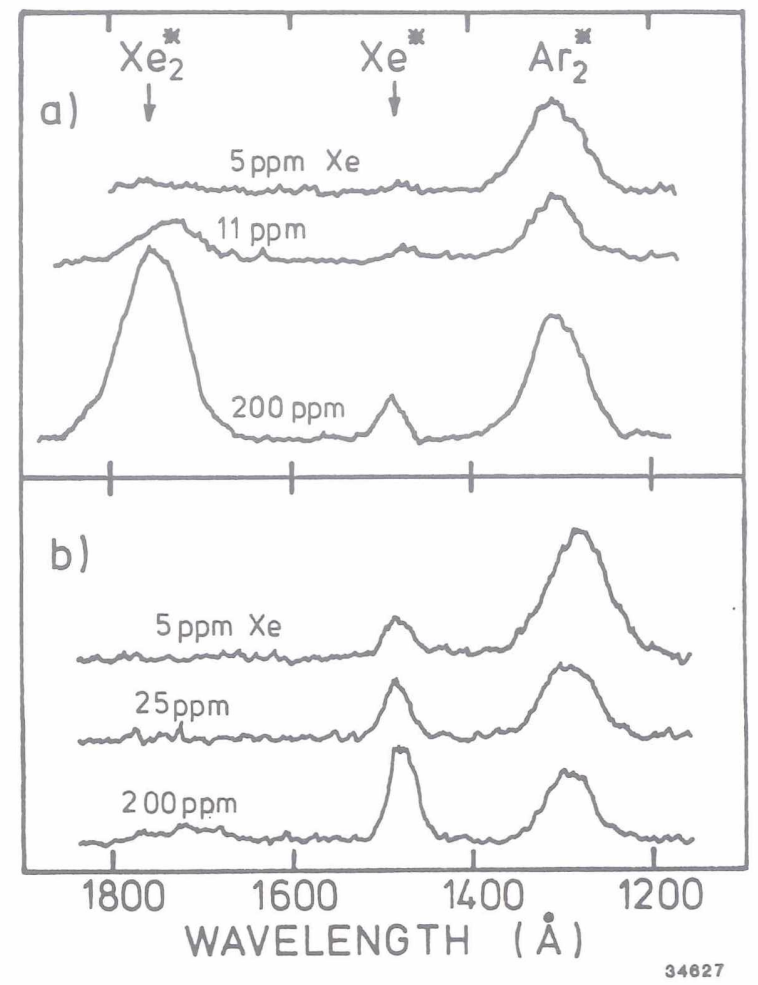

Fig. 32 Emission spectra of Xenon doped liquid argon at $87 \mathrm{~K}$ (a) and Xenon doped solid argon at $80 \mathrm{~K}$ (b). (After Cheshovsky et al., ref. 68.) 


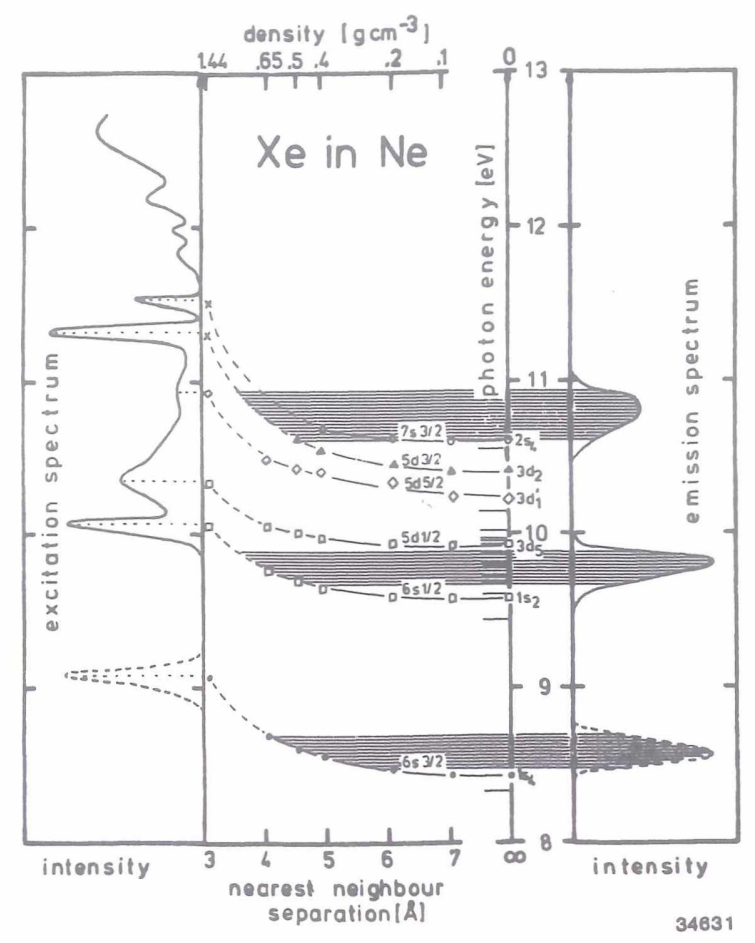

Fig. 33 Dependence of the energy position of the lowest states of Xe atoms on the density of surrounding Ne atoms. The density is expressed by the mean nearest neighbor separation between an $\mathrm{Xe}$ atom and surrounding Ne atoms. The Ne density ranges from 0 through liquid to the solid phase. For solid $\mathrm{Ne}$ (density 1,44 $\left\{\mathrm{g} \mathrm{cm}^{-3}\right\} \hat{=} .13 \AA$ ) the maxima of the excitation spectra (lefthand side) have been included. The density around relaxed $\mathrm{Xe}$ atoms follows from a comparison with the emission spectra (righthand side, horizontal lines). (From Hahn and Schwentner, ref. 70). 


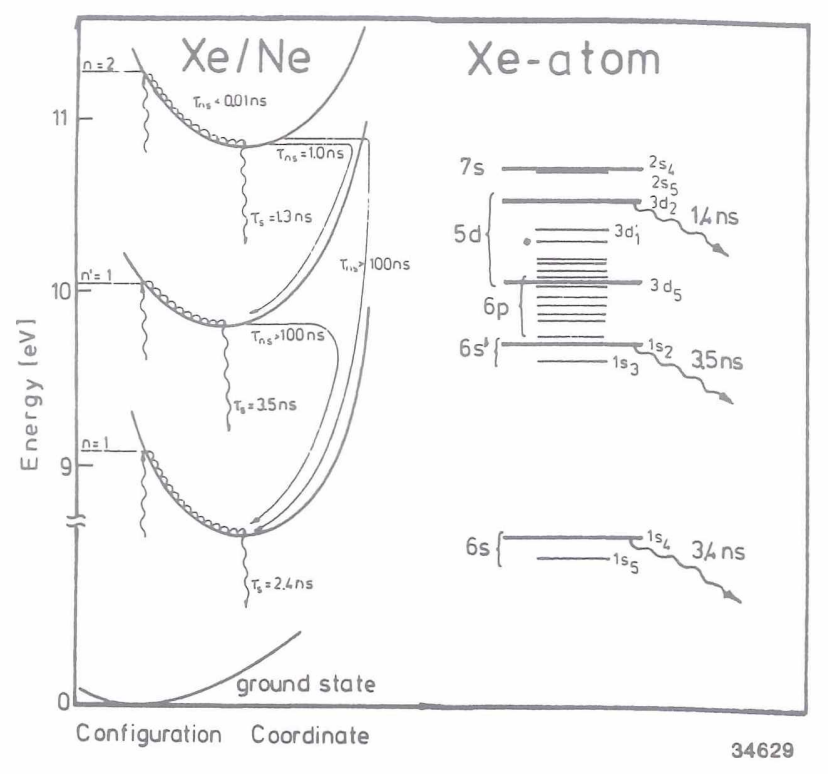

Fig. 34 Left part: experimental radiative $(\stackrel{m)}{)}$ ) and norradiative $(\longrightarrow)$ electronic relaxation pathways and time constants (in nanoseconds) for $\mathrm{Xe}$ in $\mathrm{Ne}$ in a configuration coordinate diagram. The excitation channels $n=1, n^{\prime}=1$ and $n=2$ and the matrix relaxation (mmm) are included. Right part: atomic energy levels for Xe with some lifetimes for radiative transitions to the ground state.

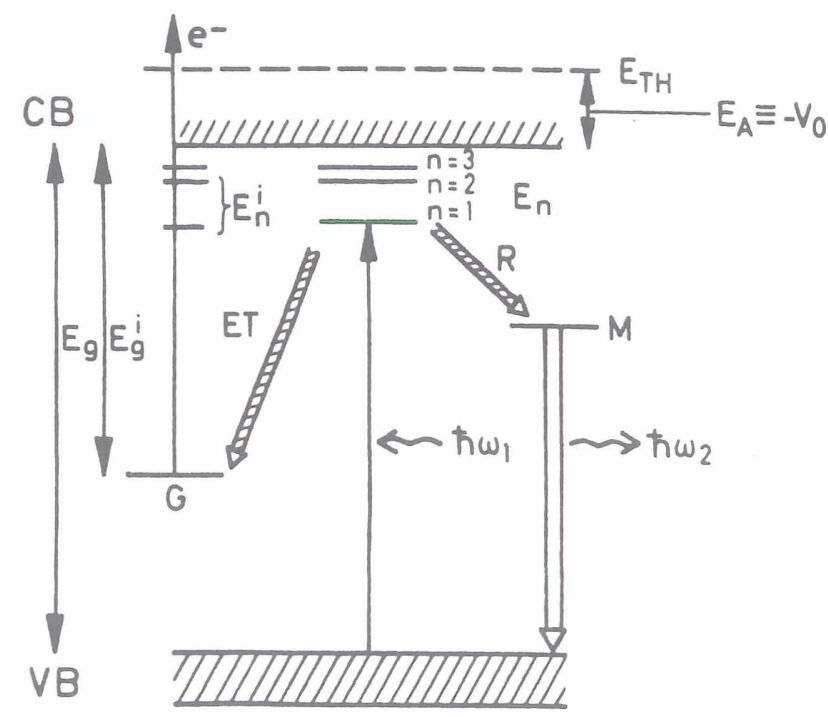

Fig. 35 Schematic energy levels scheme for impurity photoemission from doped solid rare gases. 


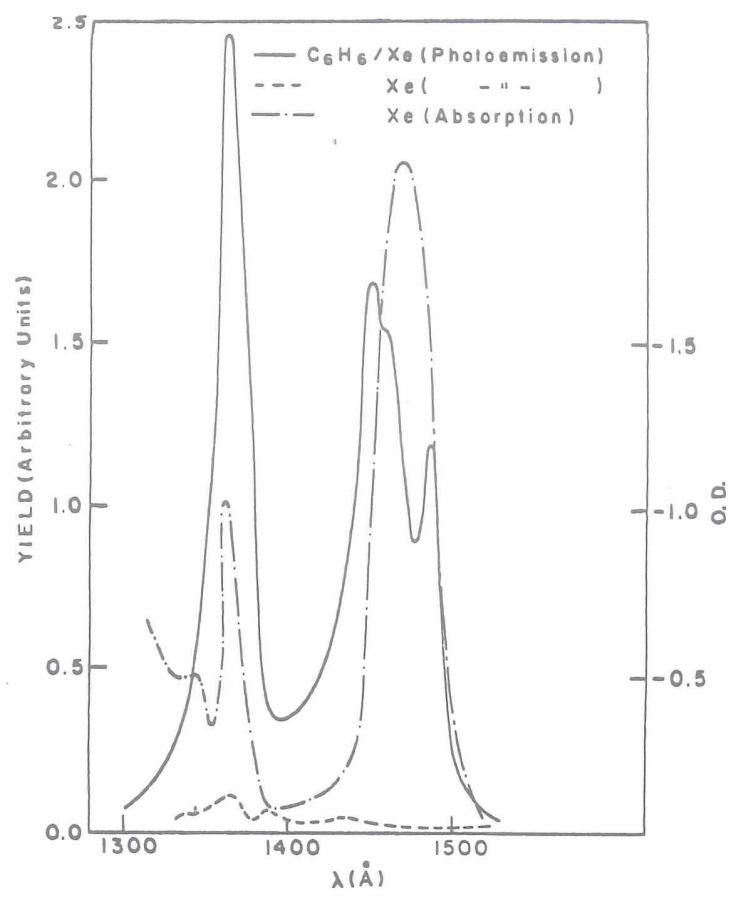

Fig. 36 Photoemission of benzene impurity (1\%) in solid Xe (reference 58). 


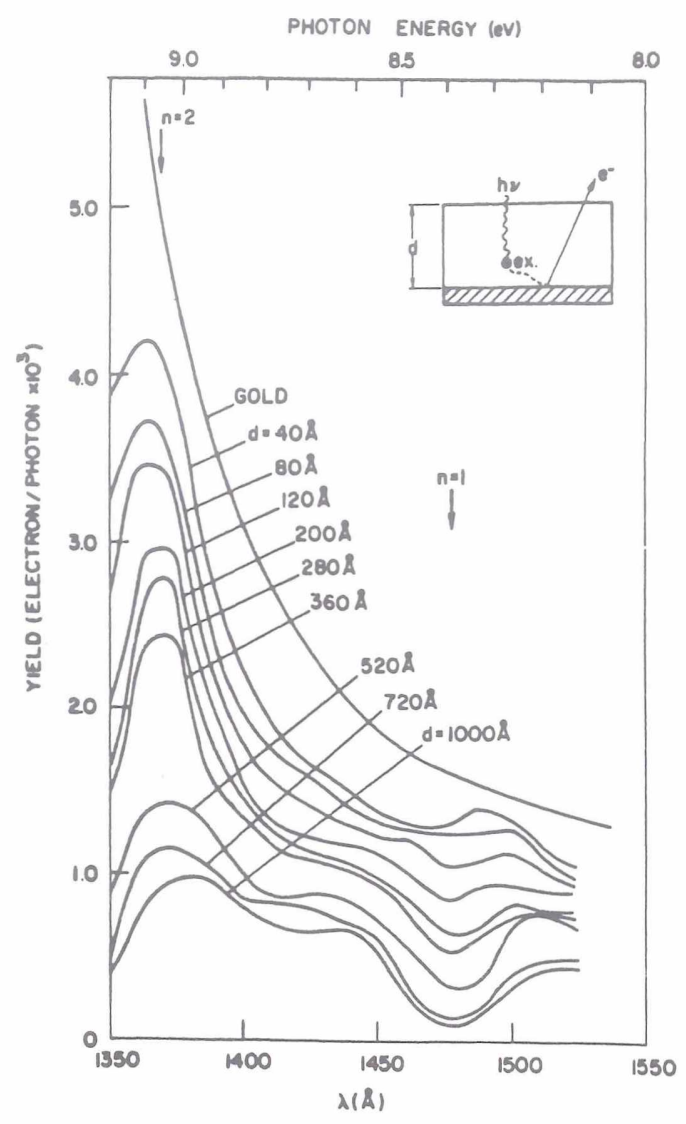

Fig. 37 Experimental photoemission yields $Y=i / I_{0}$ (where $I_{0}$ is the incident light intensity) from solid Xe deposited on the gold emitter electrode for various values of the Xe film thickness. The yield from the gold electrode (which is mounted on the LiF substrate) is also shown for comparison. The positions of the $n=1\left({ }^{2} P_{3 / 2}\right)$ and $n=2\left({ }^{2} P_{3 / 2}\right)$ excitons of solid $x e$ are marked by arrows. The upper insert portrays schematically the extrinsic photoemission mechanism via "free" exciton diffusion to the gold followed by electron ejection from the electrode (reference 71 ). 


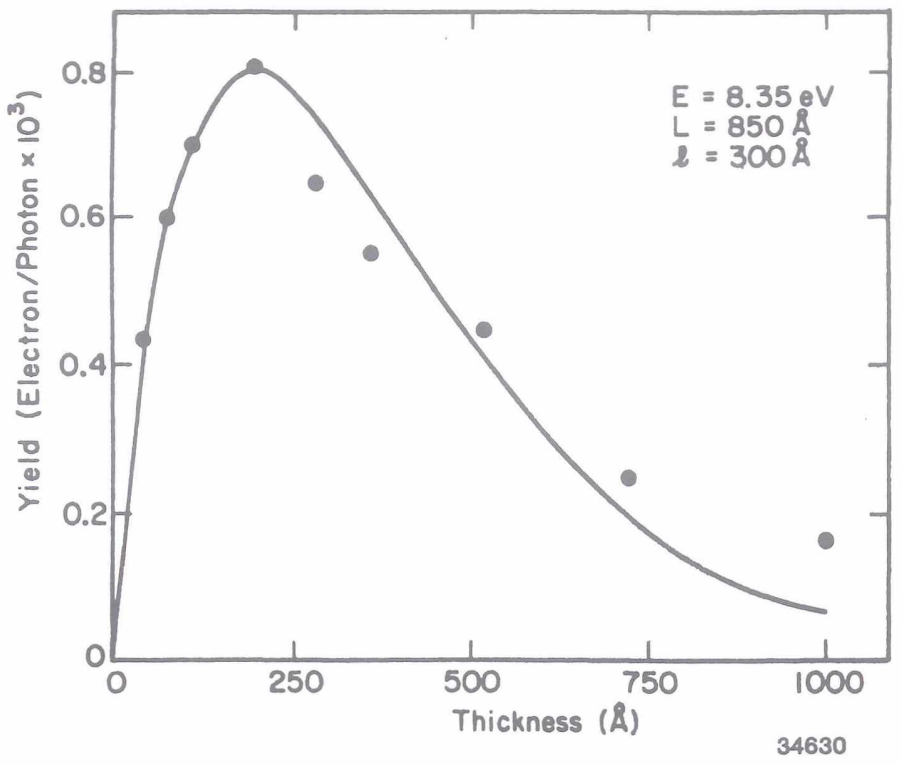

Fig. 38 The thickness dependence of the photoemission yield from Xe filris on a gold substrate. L is the electron escape length for solid Xe (ref. 71).

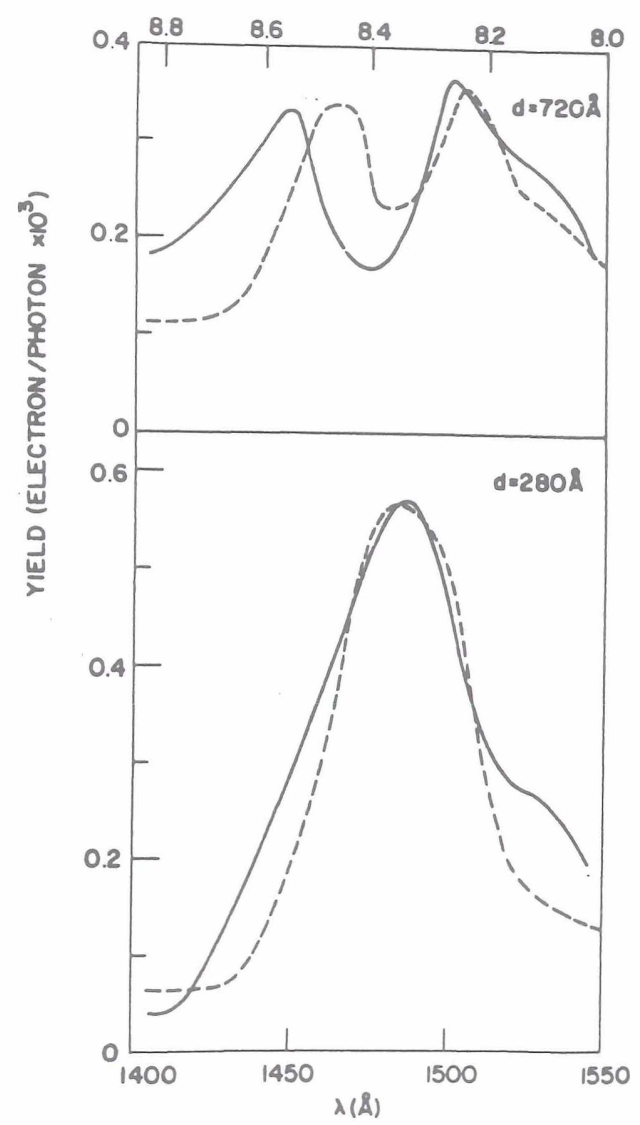

Fig. 39 The energy dependence of extrinsic photoemission from solid $\mathrm{Xe}$ on a gold substrate at two values of the film thickness. Solid lines correspond to experimental data, while dashed lines are calculated with $\ell=300 \AA$ (reference 71$)$. 
